\title{
Synthesis of 5-arylidine amino-1,3,4-thiadiazol-2-[( $N$-substituted benzyol)]sulphonamides endowed with potent antioxidants and anticancer activity induces growth inhibition in HEK293, BT474 and NCI-H226 cells
}

\author{
Mahavir Chhajed • Anil Kumar Shrivastava • \\ Vijay Taile
}

Received: 19 August 2013/ Accepted: 11 December 2013/Published online: 27 December 2013

(C) The Author(s) 2013. This article is published with open access at Springerlink.com

\begin{abstract}
A series of imines 5-amino-1,3,4-thiadiazol-2$[(N$-substituted benzyol $)]$ sulphonamide derivatives were synthesized from various aromatic aldehydes and substituted with benzoyl acetazolamides under different reaction conditions and were evaluated for their antioxidant and free radical scavenging, antimitotic activity by Allium cepa meristem root model and cytotoxicity activity against HEK 293 (human epidermal kidney cell line), BT474 (breast cancer cell line) and NCI-H226 (lung cancer cell line) by MTT assay. Some of the synthesized compounds showed moderately potent cytotoxicity compared to indisulam.
\end{abstract}

Keywords 1,3,4-Thiadiazole $\cdot$ Antimitotic ·

Antioxidants · Cytotoxicity $\cdot$ MTT assay

\section{Introduction}

The rapid spread of cancer has sparked an intense worldwide search for new compounds, which may be used in designing anticancer drugs. The search of more effective anticancer agent has focused to a large extent on the design of molecules capable of recognizing and binding to target

M. Chhajed ( $\square)$

Department of Pharmaceutical Chemistry, Suresh Gyan Vihar University, Mahal Jagat Pura, Jaipur, India

e-mail: mahavirchhajed@rediffmail.com

\section{A. K. Shrivastava}

Nandini Nagar Mahavidyalaya College of Pharmacy,

Nawabganj, Gonda, Uttar Pradesh, India

V. Taile

Department of Chemistry, RTM Nagpur University, Nagpur, India
DNA base sequences. Development of anticancer drugs with fewer or no side effects is important for the treatment for cancer. The search for such potential anticancer drugs has led to the discovery of synthetic small molecules with anti-carcinogenic activity and limited harmful side effects particularly with respect to the immune system. Research in this area is expanding rapidly, and some promising leads have emerged. Heterocyclic moieties can be found in a large number of compounds, which display biological activity. The biological activity of the compounds is mainly dependent on their molecular structures (Salimon et al., 2010). A vast number of 1,3,4-thiadiazoles have been reported as potential pharmacologically active compounds with antimicrobial (Patil and Biradar, 2001; Zamani et al., 2004; Sharma et al., 2006), antiviral (Pandey et al., 2004), antitubercular (Oruc et al., 2004; Desai et al., 1984), anticonvulsant (Shrivastava et al., 1999; Kumar et al., 2003; Gupta et al., 2008; Stillings et al., 1986; Jatav et al., 2008), CNS depressant (Jatav et al., 2008), hypoglycaemic (Hanna et al., 1995; Pattan et al., 2009), anti-inflammatory (Sharma et al., 2008; Varandas et al., 2005) and anticancer (Noolvi et al., 2011; Kumar et al., 2010) properties. At the same time, the 1,3,4-thiadiazole fragment appears in a number of clinically used drugs such as acetazolamide; methazolamide; butazolamide (diuretic); sulfamethiazole (antibacterial); cefazolin, cefazedone (antibiotic); atibeprone (anti-depressant); glybuthiazole, glybuzole (antidiabetic); and tebuthiuron (insecticide) (Wilson and Gisvold, 1991; Abrahum, 2003; Supran et al., 2003).

Schiff bases, contain an azomethine group, derived from aromatic aldehydes and aromatic amines, have potential for both chemical and biological activities (Dhar and Taploo, 1982; Pacheco et al., 1970). This is due to the presence of carbon-nitrogen double bond having potential receptorbinding ability. Schiff bases are also one of the intensively 
investigated classes of aromatic and heteroaromatic compounds. This class of compounds showed a variety of applications ranging from anticancer (Sharma et al., 1998; Kuzmin et al., 2005), antibacterial (More et al., 2002; Vaghasiya et al., 2004), diuretic (Supran et al., 1996), antifungal (Manrao et al., 1982, 1995, 2001) and antiparasitic activity (Rathelot et al., 2002). They have also medicinal importance and are used in drug design due to their activity against a wide range of organisms (Khan et al., 2002; Verma et al., 2004). Schiff bases are used as substrates in the preparation of a number of industrially and biologically active compounds via closure, cycloaddition and replacement reactions (Taggi et al., 2002).

Sulphonamides are a significant class of compounds in medicinal and pharmaceutical chemistry with several biological applications (Tilles, 2001; Slatore and Tilles, 2004; Brackett et al., 2004; Harrison, 1994; Eroglu, 2008).

There are many connections between carbonic anhydrase (CA) and cancer (Supuran, 2008; Supuran and Scozzafava, 2000; Pastorek et al., 1994; Pastorekova et al., 1997; Chegwidden et al., 2001). It is well known that some CA isozymes are predominantly found in cancer cells and are lacking from their normal counterparts (Pastorek et al., 1994; Pastorekova et al., 1997; Chegwidden et al., 2001), and these are two transmembrane isozymes CA IX and CA XII. Isozyme CA XIV was the last one to be discovered among the $15 \mathrm{CA}$ isoforms of this widespread metalloprotein known up to now in human (Supuran et al., 2004). Kaunisto et al. (2002) and Parkkila et al., $(2001,2002)$ revealed CA XIV distribution in the human body as well as potential physiological/pathological roles. It has been observed that hCA XIV is highly abundant in the brain, kidney, colon, small intestine, urinary bladder, liver and spinal cord (Kaunisto et al., 2002; Parkkila et al., 2001, 2002; Fujikawa-Adachi et al., 1999; Ashida et al., 2002). Similar to isozymes CA IX and CA XII, CA XIV is a transmembrane protein with the active site oriented extracellularly, but unlike the first two proteins, isozyme XIV is not associated with tumour cells (Pastorek et al., 1994; Kaunisto et al., 2002; Parkkila et al., 2001, 2002; Ashida et al., 2002). Membrane-associated human carbonic anhydrase (hCAs) isozymes IX, XII and XIV (Fujikawa-Adachi et al., 1999; Tureci et al., 1998) like other hCAs regulate $\mathrm{pH}$ and carbon dioxide $\left(\mathrm{CO}_{2}\right)$-bicarbonate anion $\left(\mathrm{HCO}_{3}\right)$ homoeostasis, through the catalysis of the reversible hydration of $\mathrm{CO}_{2}$ to give $\mathrm{HCO}_{3}$ and proton $(\mathrm{Hp})$. The expression level of isozymes hCA IX and XII is elevated in response to hypoxia, and research on the involvement of these isozymes in cancer has progressed considerably in recent years, particularly for hCA IX (Tureci et al., 1998; Wykoff et al., 2000; Parkkila et al., 2000; Svastova et al., 2004; Cecchi et al., 2005). It has been confirmed that hCA IX is a high-activity CA isozyme responsible for the extracellular acidification $(\mathrm{pHe})$ of the tumour microenvironment. Multiple downstream effects of this reduced $\mathrm{pHe}$ are associated with tumour progression and poor prognosis (Parkkila et al., 2000; Svastova et al., 2004). Aromatic sulphonamide compounds have been shown to reverse the effect of tumour acidification, to inhibit the growth of cancer cells and to suppress tumour invasion mediated by these CAs (Tureci et al., 1998; Wykoff et al., 2000; Parkkila et al., 2000; Svastova et al., 2004; Cecchi et al., 2005; Brzozowski et al., 2010).

Thus, the data from these many physiological studies appear to have identified a CA-mediated, hypoxic tumourspecific pathway. This provides firm grounds for exploring the effects of this class of compounds as a novel approach to discriminate between healthy cells and cancerous cells, specifically targeting hypoxic tissues, an attractive attribute that is lacking in many existing cancer therapies (Minchinton and Tannock 2006; Kamb, 2005).

These findings prompted us to the synthesis of 5-arylidine amino-1,3,4-thiadiazol-2-[( $N$-benzoyl $)]$ sulphonamide derivatives $(\mathbf{9 a}-\mathbf{j})$ from carbonic anhydrase inhibitor drug acetazolamide. The synthesized compounds reported previously (Chhajed et al., 2007, 2013), such as 5-amino-1,3,4-thiadiazol-2-[N-(substituted benzoyl)]sulphonamide $\quad(\mathbf{4 a -}-\mathbf{g})$, 5-(4-acetamido phenyl sulphonamido)-1,3,4-thiadiazol-2[N-(substituted benzoyl)]sulphonamide (6a-g), and 5-(4amino phenyl sulphonamido)-1,3,4-thiadiazol-2-[N-(substituted benzoyl)]sulphonamide (7a-g) from acetazolamide by modified Schotten-Bauman synthesis method, and compounds $(\mathbf{9} \mathbf{a}-\mathbf{j})$ reported herein are evaluated for anticancer activity, having better therapeutic index for free radical scavenging, antimitotic activity and in vitro cytotoxic activity by MTT assay for establishing their possible therapeutic value. The synthesized molecules have been characterized by various techniques such as NMR, FTIR and LCMS.

\section{Results and discussion}

\section{Chemistry}

5-Amino-1,3,4-thiadiazol-2-[ $N$-(substituted benzoyl)]sulphonamides $(\mathbf{4 a} \mathbf{a}-\mathbf{g})$ were prepared by hydrolysis of the benzoylated acetazolamides $(\mathbf{3 a}-\mathbf{g})$, which was prepared from the acetazolamide (1) by benzoylation with substituted benzoyl chlorides (2a-g). Compound (4) was refluxed with substituted aromatic aldehydes $(\mathbf{8 a}-\mathbf{j})$ using concentrated sulphuric acid as a catalyst to obtain the Schiff bases (Scheme 1).

The FT-IR spectra showed stretching at 3,485-3,265 $\mathrm{cm}^{-1}$ (sulphonamide $\mathrm{N}-\mathrm{H}), 3,037.4(\mathrm{Ar} \mathrm{C}-\mathrm{H}), 1,676-1,645(\mathrm{C}=\mathrm{O})$, $1,625-1,594 \mathrm{~cm}^{-1} \quad(\mathrm{C}=\mathrm{N}), \quad 1,517-1,530.9 \quad(\mathrm{Ar} \quad \mathrm{C}-\mathrm{C})$, $1,270 \mathrm{~cm}^{-1} \quad(\mathrm{C}-\mathrm{N}), \quad 1,177-1,125 \mathrm{~cm}^{-1}$ (sulphonamide), $1,128-1,030 \mathrm{~cm}^{-1}(\mathrm{~S}=\mathrm{O})$ and $756-662 \mathrm{~cm}^{-1}$ (thiadiazole $\mathrm{C}-\mathrm{S})$. The ${ }^{1} \mathrm{H}-\mathrm{NMR}$ spectra of all compounds indicated 
<smiles>CC(=O)Nc1nnc(S(N)(=O)=O)s1</smiles>

(1)<smiles>[R]c1ccc(C(=O)Cl)cc1</smiles>

$(2 a-g)$<smiles>[R]c1ccc(C(=O)NS(=O)(=O)c2nnc(NC(C)=O)s2)cc1</smiles>

$\mathrm{O}$

(3a-g) $10 \% \mathrm{NaOH}$

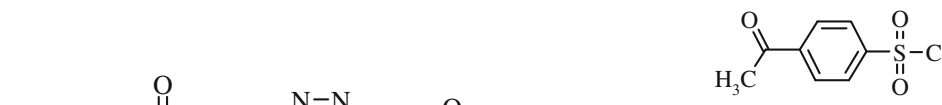

(5)<smiles>[R]c1ccc(C(=O)NS(=O)(=O)c2nnc(N)s2)cc1</smiles>

(6a-g)

ydrolysis

$\downarrow$

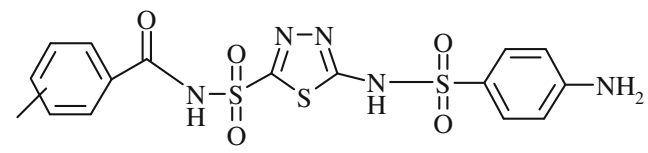

$\mathrm{R}^{1}$

$$
\text { (7a-g) }
$$

\begin{tabular}{|c|c|c|}
\hline Compd. No. & $\mathbf{R}^{1}$ & Ar \\
\hline $9 \mathrm{a}$ & $\mathrm{H}$ & $\mathrm{C}_{6} \mathrm{H}_{5}$ \\
\hline $9 b$ & $\mathrm{H}$ & $p-\mathrm{ClC}_{6} \mathrm{H}_{4}$ \\
\hline $9 \mathrm{c}$ & $\mathrm{H}$ & $o-\mathrm{OCH}_{3} \mathrm{C}_{6} \mathrm{H}_{4}$ \\
\hline $9 d$ & $\mathrm{H}$ & $p-\mathrm{OCH}_{3} \mathrm{C}_{6} \mathrm{H}_{4}$ \\
\hline $9 \mathrm{e}$ & $\mathrm{H}$ & $p-\mathrm{OH} \mathrm{C} \mathrm{H}_{4}$ \\
\hline 9f & $\mathrm{H}$ & $o-\mathrm{OH} \mathrm{C} \mathrm{H}_{4}$ \\
\hline $9 g$ & $\mathrm{H}$ & $m-\mathrm{OCH}_{3}, p-\mathrm{OH} \mathrm{C} \mathrm{C}_{3}$ \\
\hline $9 \mathrm{~h}$ & $\mathrm{H}$ & $p-\mathrm{N}\left(\mathrm{CH}_{3}\right)_{2} \mathrm{C}_{6} \mathrm{H}_{4}$ \\
\hline $9 \mathrm{i}$ & $\mathrm{H}$ & $p-\mathrm{NO}_{2} \mathrm{C}_{6} \mathrm{H}_{4}$ \\
\hline $9 j$ & $\mathrm{H}$ & \\
\hline
\end{tabular}

Scheme 1 Synthesis of 5-amino-1,3,4-thiadiazol-2-[N-(substituted benzoyl)]sulphonamide (4a-g), 5-(4-acetamido phenyl sulphonamido)-1,3,4-thiadiazole-2-[ $N$-(substituted benzoyl)]sulphonamide (6a-

expected peaks in the region of 1.249-1.254 $\delta \mathrm{ppm}(\mathrm{s}, \mathrm{Ar}-$ $\left.\mathrm{SO}_{2} \mathrm{NH}\right), 3.569-4.116 \delta \mathrm{ppm}$ (s, Schiff base $\left.\mathrm{CH}=\mathrm{N}\right)$ and 8.24-8.523 $\delta$ ppm (s, amide $\mathrm{C}(=\mathrm{O}) \mathrm{N}-\mathrm{H})$, while multiplets of aromatic ring are in the range of 6.6-8.2 $\delta \mathrm{ppm}$. Thin-layer chromatography (TLC) was run throughout the reaction to optimize the reaction for purity and completion. g), 5-(4-amino phenyl sulphonamido)-1,3,4-thiadiazole-2-[ $N$-(substituted benzoyl)]sulphonamide (7a-g), and 5-arylidine amino-1,3,4thiadiazol-2-[ $N$-(substituted benzoyl)] sulphonamide $(\mathbf{9 a}-\mathbf{j})$

Pharmacological evaluation

Antioxidant and free radical scavenging activity

$\mathrm{ABTS}^{+}$radical, lipid peroxidation, DPPH radical, superoxide anion and nitric oxide anion radical scavenging 
Table 1 Comparative $\mathrm{IC}_{50}$ inhibitory concentration of synthesized compounds and standards against different free radicals

\begin{tabular}{|c|c|c|c|c|c|}
\hline \multirow[t]{2}{*}{ Compound no. } & \multicolumn{5}{|c|}{$\mathrm{IC}_{50}$ inhibitory concentration $(\mathrm{nM} / \mathrm{mL})^{\mathrm{a}}$} \\
\hline & $\mathrm{ABTS}^{+}$radical $^{\mathrm{b}}$ & Lipid peroxidation $^{\mathrm{c}}$ & DPPH radical ${ }^{\mathrm{d}}$ & Superoxide anion $^{\mathrm{e}}$ & Nitric oxide radical \\
\hline $9 a$ & $\begin{array}{l}73.30 \pm 7.05^{*} \\
{[4.07]}\end{array}$ & $\begin{array}{l}121.63 \pm 18.60 \\
{[10.74]}\end{array}$ & $\begin{array}{l}134.07 \pm 12.90^{*} \\
{[22.34]}\end{array}$ & $\begin{array}{l}151.89 \pm 14.42 * \\
{[24.97]}\end{array}$ & $\begin{array}{l}103.67 \pm 7.50^{*} \\
{[12.99]}\end{array}$ \\
\hline $9 b$ & $\begin{array}{l}93.30 \pm 10.67 * \\
{[6.16]}\end{array}$ & $\begin{array}{l}133.02 \pm 11.53 * \\
{[6.65]}\end{array}$ & $\begin{array}{l}88.19 \pm 11.09 * \\
{[6.40]}\end{array}$ & $\begin{array}{l}76.31 \pm 11.80^{*} \\
{[6.81]}\end{array}$ & $\begin{array}{l}52.57 \pm 16.73^{*} \\
{[9.66]}\end{array}$ \\
\hline $9 c$ & $\begin{array}{l}196.17 \pm 16.60 * \\
{[9.58]}\end{array}$ & $\begin{array}{l}101.78 \pm 14.51 * * \\
{[8.38]}\end{array}$ & $\begin{array}{l}41.27 \pm 4.23 * * \\
{[2.44]}\end{array}$ & $\begin{array}{l}128.09 \pm 21.74 * \\
{[12.55]}\end{array}$ & $\begin{array}{l}81.90 \pm 10.44 * \\
{[6.02]}\end{array}$ \\
\hline 9d & $\begin{array}{l}55.61 \pm 6.98 * \\
{[4.03]}\end{array}$ & $\begin{array}{l}164.49 \pm 14.56^{*} \\
{[8.41]}\end{array}$ & $\begin{array}{l}63.56 \pm 8.35^{* *} \\
{[4.82]}\end{array}$ & $\begin{array}{l}74.52 \pm 8.3^{*} \\
{[4.79]}\end{array}$ & $\begin{array}{l}53.03 \pm 6.74 * \\
{[3.89]}\end{array}$ \\
\hline $9 e$ & $\begin{array}{l}47.89 \pm 9.90 * \\
{[5.72]}\end{array}$ & $\begin{array}{l}134.34 \pm 14.70^{* *} \\
{[8.49]}\end{array}$ & $\begin{array}{l}107.28 \pm 18.13 * * \\
{[10.46]}\end{array}$ & $\begin{array}{l}135.52 \pm 22.55^{*} \\
{[13.02]}\end{array}$ & $\begin{array}{l}155.21 \pm 17.64 * \\
{[10.19]}\end{array}$ \\
\hline 9f & $\begin{array}{l}207.14 \pm 17.41 * \\
{[10.05]}\end{array}$ & $\begin{array}{l}203.74 \pm 20.11 * * \\
{[11.61]}\end{array}$ & $\begin{array}{l}80.63 \pm 11.38 * * \\
{[6.57]}\end{array}$ & $\begin{array}{l}36.6604 \pm 14.39 * \\
{[8.31]}\end{array}$ & $\begin{array}{l}38.00 \pm 11.77 * \\
{[6.79]}\end{array}$ \\
\hline Std & $\begin{array}{l}84.54 \pm 9.39 * \\
{[5.42]}\end{array}$ & $\begin{array}{l}150.12 \pm 16.93 * * \\
{[9.77]}\end{array}$ & $\begin{array}{l}187.20 \pm 35.38^{*} \\
{[19.96]}\end{array}$ & $\begin{array}{l}171.36 \pm 9.10^{* *} \\
{[5.25]}\end{array}$ & $\begin{array}{l}73.67 \pm 9.44 * \\
{[5.45]}\end{array}$ \\
\hline
\end{tabular}

$* P<0.05 ; * * P<0.01$

${ }^{\text {a }} \mathrm{IC}_{50}$ value reported as Conc. \pm SD [SEM]; SEM of three independent experiments performed in duplicate

b Standard used was trolox

c Standard used was ascorbic acid

${ }^{\mathrm{d}}$ Standard used was ascorbic acid

e Standard used was catechin

${ }^{\mathrm{f}}$ Standard used was curcumin

activity has been used as a quick and reliable parameter to assess the in vitro antioxidant activity. Each method relates to the generation of a different radical, acting through a variety of mechanisms and the measurement of a range of end points at a fixed time point or over a range (Miller and Rice-Evans, 1994, 1996). The different concentrations of the synthesized compounds showed antioxidant activities in a dose-dependent manner. Comparative $\mathrm{IC}_{50}(\mathrm{nM} / \mathrm{mL})$ inhibitory concentrations of synthesized compounds against different free radicals are reported in Table 1. All the tested compounds showed statistically significant $(P<0.05) \mathrm{IC}_{50}$ values. Among the tested compounds, $(\mathbf{9 c})$ is the most potent compound and had lowest $\mathrm{IC}_{50}(\mathrm{nM} / \mathrm{mL})$ value against DPPH radical, nitric oxide anion and lipid peroxidation, while (9e) and (9f) showed maximum potency against $A B T S^{+}$radical and superoxide anion radical, respectively. The study also indicates that the compounds (9c), (9d) and (9f) showed the smaller $\mathrm{IC}_{50}$ $(\mathrm{nM} / \mathrm{mL})$ values even than respective standards, indicating that these compounds are more potent than the standard, and reveals that the electron-donating functional group like $-\mathrm{OCH}_{3}$ (9c and 9d) or the functional group like $-\mathrm{OH}$ having the ability to bind with free radical (9f) is responsible for the potency.
Antimitotic activity

The levels of the physicochemical parameters of Allium cepa (root number and root length) were recorded after treatment with various drugs at 0,48 and $72 \mathrm{~h}$ and found to cause significant inhibition in the growth of roots in comparison with negative control and positive control. From the observations, it has been revealed that average root length in (9f) treatment group was decreased significantly $(1.06 \mathrm{~cm})$ compared with that of the negative control $(3.93 \mathrm{~cm})$ after $72 \mathrm{~h}$ of treatment. The root morphology was nearly normal during the negative control treatment, but at positive control and synthesized compound groups, the roots morphology showed an obvious difference in its appearance in that it turned to slightly yellowish to brownish in colour. Its cytotoxic effect was evident in the form of shortening and decaying of roots, while progressive increases in root length and root numbers were observed in control group. The cytotoxic effect of tested compounds inhibits root growth and mitosis to a significant extent. The compound 9f showed lowest mitotic index $(0.41 \%)$ with highest activity among all the treatment groups, and it was also observed that the number of nondividing cells increased in all treatment groups other than 
Table 2 Mitotic index and chromosome and mitotic aberrations in the root meristem cells of Allium cepa after the synthesized compounds treatment

\begin{tabular}{|c|c|c|c|c|c|c|c|}
\hline $\begin{array}{l}\text { Treatment } \\
\text { groups }\end{array}$ & Dose & $\begin{array}{l}\mathrm{MI} \\
(\%) \pm \mathrm{SEM}^{\mathrm{a}, \mathrm{b}}\end{array}$ & $\begin{array}{l}\text { Chromosome breaks } \\
(\%) \pm \mathrm{SEM}^{\mathrm{b}}\end{array}$ & $\begin{array}{l}\text { Stickiness } \\
(\%) \pm \text { SEM }^{b}\end{array}$ & $\begin{array}{l}\text { Polar deviations } \\
(\%) \pm \mathrm{SEM}^{\mathrm{b}}\end{array}$ & $\begin{array}{l}\text { Aberrant cells } \\
(\%) \pm \text { SEM }^{\mathrm{b}}\end{array}$ & $\begin{array}{l}\mathrm{MNC} \\
(\% 0) \pm \mathrm{SEM}^{\mathrm{t}}\end{array}$ \\
\hline $\mathrm{NC}^{\mathrm{c}}$ & - & $6.22 \pm 0.32$ & - & $0.92 \pm 0.32$ & $6.89 \pm 1.32$ & $10.12 \pm 1.58$ & $0.35 \pm 0.12$ \\
\hline $\mathrm{PC}^{\mathrm{d}}$ & $2 \times 10^{-2} \mathrm{M}$ & $1.86 \pm 0.23$ & - & $36.31 \pm 9.84$ & $12.36 \pm 3.36$ & $43.20 \pm 7.10$ & $0.59 \pm 0.09$ \\
\hline $9 a$ & $1 \mathrm{mg} / \mathrm{mL}$ & $3.22 \pm 0.16$ & $6.22 \pm 1.02$ & $6.64 \pm 2.38$ & $8.62 \pm 2.16$ & $19.28 \pm 5.22$ & $0.34 \pm 0.15$ \\
\hline $9 b$ & $1 \mathrm{mg} / \mathrm{mL}$ & $2.77 \pm 0.19$ & $3.36 \pm 0.57$ & $9.12 \pm 1.33$ & $7.32 \pm 1.24$ & $24.64 \pm 7.01$ & $0.42 \pm 0.18$ \\
\hline $9 c$ & $1 \mathrm{mg} / \mathrm{mL}$ & $0.53 \pm 0.03$ & - & $28.04 \pm 6.34$ & $7.22 \pm 2.61$ & $38.54 \pm 8.18$ & $0.36 \pm 0.14$ \\
\hline 9d & $1 \mathrm{mg} / \mathrm{mL}$ & $2.34 \pm 0.19$ & $0.96 \pm 0.46$ & $14.48 \pm 2.52$ & $9.15 \pm 6.92$ & $25.33 \pm 9.42$ & $0.51 \pm 0.17$ \\
\hline $9 e$ & $1 \mathrm{mg} / \mathrm{mL}$ & $1.27 \pm 0.11$ & $2.72 \pm 0.94$ & $9.88 \pm 1.46$ & $8.41 \pm 1.35$ & $26.74 \pm 6.56$ & $0.21 \pm 0.06$ \\
\hline 9f & $1 \mathrm{mg} / \mathrm{mL}$ & $0.91 \pm 0.13$ & $1.47 \pm 0.13$ & $21.96 \pm 7.22$ & $7.33 \pm 2.52$ & $33.41 \pm 9.47$ & $0.39 \pm 0.20$ \\
\hline $9 \mathrm{~g}$ & $1 \mathrm{mg} / \mathrm{mL}$ & $0.41 \pm 0.04$ & - & $32.24 \pm 6.92$ & $10.26 \pm 2.13$ & $40.48 \pm 12.94$ & $0.48 \pm 0.32$ \\
\hline $9 h$ & $1 \mathrm{mg} / \mathrm{mL}$ & $1.07 \pm 0.13$ & $2.43 \pm 0.67$ & $16.50 \pm 3.23$ & $8.91 \pm 1.56$ & $29.83 \pm 5.03$ & $0.31 \pm 0.14$ \\
\hline $9 \mathbf{i}$ & $1 \mathrm{mg} / \mathrm{mL}$ & $3.07 \pm 0.22$ & $7.33 \pm 2.06$ & $7.35 \pm 2.06$ & $6.57 \pm 1.33$ & $22.41 \pm 6.18$ & $0.61 \pm 0.15$ \\
\hline
\end{tabular}

a The MI values indicates that lower the MI value higher the activity

b All the values are expressed as mean \pm SEM "data are the mean, SEM of 3 independent experiments performed in duplicate"

${ }^{c}$ Distilled water was used as negative control (NC)

${ }^{\mathrm{d}}$ Ethyl methanesulphonate (EMS) was used as positive control (PC)

negative control. As there is no antimitotic principle in water, it was considered as negative control. Ethyl methanesulphonate (EMS) was treated as positive control treatment group and induces DNA damage by a direct mechanism, acting at various sites as a monofunctional ethylating agent of nucleotides (Budavari, 1989; Sega, 1984).

\section{Cytogenetic analysis}

With the objective of investigating the possible mechanism involved in root growth inhibition, cytogenetic analysis was performed (Angayarkanni et al., 2007; Auti et al., 2010; Pavlica et al., 2000). All the tested compounds provoked strong inhibition of the mitotic index, where a statistically significant difference in relation to the control, and the decrease in the mitotic index was positively correlated with the electron-releasing group (Table 2). Changes in chromosome and cellular morphology were observed with increasing time. Partial c-mitosis (colchicine-like mitosis) and full c-mitosis, with partially functional spindles and completely normal mitotic phases, were seen in the various cells of the same root tip between 6- and 72-h time period. Cytogenetic alterations were investigated, and the results are depicted in Table 2. All the tested compounds induced chromosome and cytological alterations in treatment groups. An analysis of chromosome aberrations showed that most of the fragments detected in the different treatments were of chromosome type. The observation of chromosome breaks showed the clastogenic effect of tested compounds. The occurrence of chromosome fragments allows observation of statistically significant differences at tested synthesized compounds. In addition to the chromosome fragments, sticky metaphase and polar deviations (wrong directions of chromosome movement) were also observed. In general, it is possible to observe an increase in different abnormalities as the nucleophilic functional group concentration increased. In Allium test, a strong toxic effect of tested compounds was observed, supported by great occurrence of sticky metaphases, leading to cellular death (mitotic index decrease). All the tested compounds produced a significant decrease in mitotic index were time dependent at the treatment of $1 \mathrm{mg} / \mathrm{mL}$. There was a statistically significant increase in total aberrant cells $(P<0.05)$ (aberrant cells include chromosome breaks, thickness and polar deviation) as compared with the negative control (Table 2); however, the highest value of aberrant cells is shown by the positive control. Statistical analysis showed that the genotoxic activities of the tested compounds induced micronuclei in the root tip meristem cells of A. cepa. Micronucleus formation in 1,000 cells per slide (\%MNC value) was also increased in tested compounds and in positive control EMS compared with negative control, which is statistically significant $(P<0.05)$.

In the light of the results obtained in the present study, these observations above may be due to the genotoxic and nucleotoxic action of the compounds or the disturbance of the formation of spindle fibres during cell division, which leads to chromosomal aberrations. Stickiness and clumping of the chromosomes were some of the most common effects of these tested compounds on the treated root tips. Stickiness usually leads to the formation of anaphase and telophase bridges, and this ends up inhibiting 
Table 3 Anticancer activity (\% cytotoxicity) and CTC $_{50}$ values of synthesized compounds on HEK 293 (human epidermal kidney cell line)

\begin{tabular}{|c|c|c|c|c|c|c|c|c|c|c|c|}
\hline \multirow[t]{3}{*}{ Treatment } & \multicolumn{10}{|c|}{$\%$ cytotoxicity $(100-\%$ cell survival $)$ of HEK 293 cell line at conc. $(\mu \mathrm{M})$} & \multirow[t]{3}{*}{$\mathrm{CTCC}_{50}(\mu \mathrm{M})^{\mathrm{a}}$} \\
\hline & \multicolumn{10}{|c|}{ Log conc. } & \\
\hline & 2.00 & 1.52 & 1.05 & 0.57 & 0.09 & -0.39 & -0.89 & -1.35 & -1.82 & -2.30 & \\
\hline $\mathbf{4 a}$ & 32.96 & 31.71 & 29.48 & 28.87 & 28.54 & 28.18 & 26.93 & 26.64 & 25.82 & 25.57 & 64.363 \\
\hline $4 \mathbf{b}$ & 65.41 & 63.14 & 62.32 & 59.72 & 58.13 & 57.56 & 53.61 & 50.42 & 47.02 & 41.45 & 0.922 \\
\hline $4 c$ & 49.12 & 47.84 & 46.53 & 42.12 & 40.66 & 39.93 & 39.10 & 38.24 & 37.87 & 36.34 & 4.563 \\
\hline $4 d$ & 48.13 & 47.57 & 47.04 & 44.62 & 42.39 & 42.08 & 40.54 & 39.42 & 38.30 & 37.27 & 10.347 \\
\hline $4 e$ & 40.20 & 40.04 & 39.12 & 38.89 & 37.12 & 35.43 & 34.75 & 34.13 & 31.57 & 30.58 & 1.8846 \\
\hline $4 f$ & 31.97 & 31.19 & 30.74 & 30.04 & 29.17 & 28.85 & 28.43 & 28.12 & 26.39 & 24.28 & 120.951 \\
\hline $4 g$ & 50.18 & 48.71 & 47.08 & 46.35 & 45.62 & 45.14 & 43.74 & 41.18 & 40.53 & 39.32 & 2.798 \\
\hline $6 \mathbf{a}$ & 35.42 & 35.16 & 34.98 & 33.56 & 32.17 & 30.14 & 29.88 & 28.19 & 26.78 & 26.51 & 97.475 \\
\hline $\mathbf{6 b}$ & 48.23 & 46.83 & 45.29 & 43.99 & 43.13 & 42.63 & 39.91 & 37.86 & 36.22 & 35.64 & 4.324 \\
\hline $6 c$ & 38.78 & 38.22 & 37.79 & 36.59 & 35.72 & 34.75 & 33.58 & 32.94 & 32.05 & 30.46 & 187.19 \\
\hline 6d & 41.30 & 40.73 & 39.29 & 38.41 & 37.16 & 36.73 & 35.94 & 35.10 & 34.80 & 33.32 & 31.793 \\
\hline $6 e$ & 54.97 & 51.16 & 49.87 & 49.15 & 47.06 & 45.27 & 43.36 & 42.66 & 41.98 & 39.12 & 3.937 \\
\hline $6 f$ & 62.43 & 59.31 & 58.65 & 54.16 & 51.24 & 49.12 & 47.20 & 45.35 & 42.21 & 39.29 & 1.122 \\
\hline $6 \mathrm{~g}$ & 31.97 & 28.73 & 26.15 & 24.22 & 20.81 & 20.09 & 18.32 & 18.01 & 16.52 & 15.14 & 6.658 \\
\hline $7 \mathbf{a}$ & 35.69 & 34.15 & 33.49 & 32.54 & 32.45 & 30.16 & 28.58 & 26.39 & 25.75 & 23.69 & 5.525 \\
\hline $7 b$ & 51.86 & 50.68 & 48.17 & 47.80 & 46.53 & 45.26 & 43.99 & 40.45 & 39.24 & 37.78 & 2.268 \\
\hline $7 c$ & 49.93 & 49.17 & 49.15 & 47.06 & 45.27 & 43.36 & 42.66 & 40.65 & 38.21 & 36.49 & 4.621 \\
\hline $7 d$ & 29.58 & 29.03 & 27.25 & 26.57 & 25.26 & 24.12 & 22.18 & 20.28 & 19.87 & 18.85 & 31.443 \\
\hline $7 e$ & 39.76 & 38.78 & 38.08 & 36.42 & 35.48 & 34.68 & 32.12 & 30.19 & 28.97 & 26.94 & 2.337 \\
\hline $7 f$ & 43.78 & 41.25 & 40.59 & 39.53 & 38.74 & 37.52 & 36.99 & 36.04 & 35.11 & 33.19 & 0.754 \\
\hline $7 \mathrm{~g}$ & 42.87 & 40.29 & 38.13 & 37.17 & 36.52 & 35.91 & 35.14 & 33.26 & 31.16 & 29.12 & 1.261 \\
\hline $9 a$ & 50.59 & 46.23 & 45.62 & 44.17 & 43.11 & 42.42 & 40.73 & 39.83 & 38.24 & 37.35 & 24.642 \\
\hline $9 b$ & 40.72 & 38.89 & 38.60 & 38.21 & 38.04 & 37.73 & 36.59 & 34.57 & 34.08 & 33.23 & 1.162 \\
\hline $9 c$ & 52.34 & 47.41 & 45.94 & 44.29 & 43.13 & 42.92 & 42.06 & 40.33 & 38.16 & 36.83 & 2.413 \\
\hline 9d & 38.89 & 38.22 & 36.31 & 35.84 & 35.51 & 34.78 & 34.75 & 33.85 & 32.57 & 30.64 & 12.77 \\
\hline $9 e$ & 39.61 & 37.65 & 34.24 & 31.41 & 30.29 & 29.81 & 28.32 & 26.59 & 26.66 & 25.27 & 16.044 \\
\hline 9f & 42.81 & 39.79 & 37.94 & 37.43 & 37.11 & 36.42 & 35.14 & 34.03 & 33.12 & 32.53 & 7.428 \\
\hline $9 \mathrm{~g}$ & 38.61 & 34.14 & 33.55 & 32.77 & 32.09 & 31.15 & 30.32 & 28.54 & 27.57 & 25.40 & 22.12 \\
\hline $9 \mathrm{~h}$ & 37.59 & 36.90 & 36.25 & 35.73 & 35.68 & 35.06 & 34.82 & 34.54 & 32.93 & 32.02 & 1.829 \\
\hline $9 \mathrm{i}$ & 43.48 & 39.51 & 38.84 & 37.19 & 37.03 & 36.69 & 36.32 & 35.12 & 34.46 & 33.04 & 41.71 \\
\hline $9 \mathbf{j}$ & 38.91 & 36.86 & 36.12 & 35.26 & 35.02 & 34.51 & 34.31 & 33.73 & 32.81 & 31.41 & 2.934 \\
\hline ISL & 69.39 & 61.24 & 57.83 & 55.37 & 52.22 & 51.07 & 50.12 & 48.56 & 46.89 & 42.28 & 0.217 \\
\hline
\end{tabular}

${ }^{\mathrm{a}} \mathrm{CTC}_{50}$ cytotoxicity concentration $(\mu \mathrm{M})$ determined experimentally

post-telophase, metaphase and cytokinesis, respectively, and thus hampering cell division.

\section{In vitro cytotoxicity activity by MTT assay method}

All the synthesized compounds prepared by Scheme I and previously reported (Chhajed et al., 2007, 2013) compounds were subjected to anticancer activity. $\mathrm{CTC}_{50}$ (cytotoxic concentration at which $50 \%$ of the cells are dead after drug exposure) determined for test and standard compound with the help of MTT assay HEK 293 (epidermal kidney cell line), BT474 (breast cancer cell line) and NCI-H226 (lung cancer) cell lines by MTT method (Freshney, 2000; Edmondson et al., 1988; Prasad et al., 2005; Chiruvella et al., 2008). The viability of control cells was designated as $100 \%$, and the others were expressed as percentage compared to the control. The results were compared with standard drug indisulam (ISL). The results demonstrated a strong dose-dependent growth inhibition in treated cell lines. It showed that different cells had a 
Table 4 Anticancer activity (\% cytotoxicity) and $\mathrm{CTC}_{50}$ values of synthesized compounds on BT474 (breast cancer cell line)

\begin{tabular}{|c|c|c|c|c|c|c|c|c|c|c|c|}
\hline \multirow[t]{4}{*}{ Treatment } & \multicolumn{10}{|c|}{$\%$ cytotoxicity $(100-\%$ cell survival $)$ of BT474 cell line at conc. $(\mu \mathrm{M})$} & \multirow[t]{4}{*}{$\mathrm{CTC}_{50}(\mu \mathrm{M})^{\mathrm{a}}$} \\
\hline & & 33.33 & 11.11 & 3.7 & 1.23 & 0.41 & 0.13 & 0.045 & 0.015 & 0.005 & \\
\hline & \multicolumn{10}{|c|}{ Log conc. } & \\
\hline & 2.00 & 1.52 & 1.05 & 0.57 & 0.09 & -0.39 & -0.89 & -1.35 & -1.82 & -2.30 & \\
\hline $4 \mathbf{a}$ & 28.73 & 28.11 & 27.45 & 26.28 & 25.92 & 25.37 & 24.64 & 23.12 & 22.64 & 20.06 & 61.336 \\
\hline $4 b$ & 26.66 & 23.31 & 22.19 & 20.47 & 19.85 & 18.14 & 17.99 & 17.37 & 16.56 & 16.18 & 5.496 \\
\hline $4 c$ & 41.35 & 40.32 & 39.37 & 38.82 & 37.56 & 36.26 & 35.55 & 34.19 & 32.11 & 30.65 & 8.743 \\
\hline $4 d$ & 32.09 & 30.34 & 29.44 & 28.10 & 27.13 & 26.82 & 26.23 & 25.34 & 24.24 & 23.19 & 1.746 \\
\hline $4 e$ & 40.37 & 38.91 & 37.21 & 36.96 & 35.73 & 33.14 & 32.29 & 31.76 & 31.02 & 30.89 & 2.798 \\
\hline $4 f$ & 59.31 & 55.26 & 52.38 & 50.12 & 48.54 & 45.32 & 43.76 & 41.28 & 39.05 & 37.60 & 1.561 \\
\hline $4 g$ & 38.22 & 37.84 & 36.21 & 35.19 & 34.87 & 34.15 & 33.18 & 32.07 & 31.45 & 30.59 & 2.346 \\
\hline $6 a$ & 32.69 & 32.09 & 31.26 & 30.89 & 30.38 & 29.83 & 28.61 & 27.96 & 27.18 & 26.01 & 11.147 \\
\hline $\mathbf{6 b}$ & 31.97 & 30.32 & 29.34 & 28.72 & 28.14 & 27.13 & 26.25 & 25.78 & 25.06 & 24.32 & 3.656 \\
\hline $6 c$ & 39.44 & 38.21 & 37.91 & 37.09 & 36.69 & 35.37 & 34.95 & 34.13 & 33.27 & 33.11 & 11.552 \\
\hline $6 d$ & 33.85 & 33.29 & 32.92 & 32.11 & 31.02 & 30.56 & 29.44 & 28.93 & 27.72 & 26.34 & 127.620 \\
\hline $6 e$ & 37.27 & 34.77 & 32.45 & 31.08 & 30.13 & 29.38 & 28.67 & 28.11 & 28.01 & 27.14 & 2.418 \\
\hline 6f & 50.81 & 45.31 & 42.19 & 40.62 & 37.19 & 35.84 & 33.41 & 32.15 & 30.07 & 29.13 & 1.007 \\
\hline $6 \mathrm{~g}$ & 46.38 & 44.19 & 42.44 & 39.51 & 38.20 & 37.56 & 34.12 & 33.86 & 32.75 & 30.46 & 1.028 \\
\hline $7 \mathbf{a}$ & 46.32 & 43.67 & 41.82 & 40.72 & 39.54 & 38.21 & 37.77 & 36.69 & 34.95 & 34.13 & 9.215 \\
\hline $7 b$ & 36.61 & 35.52 & 34.59 & 33.33 & 32.16 & 31.36 & 30.24 & 29.47 & 28.13 & 27.42 & 1.884 \\
\hline $7 c$ & 27.87 & 26.43 & 25.71 & 24.22 & 22.81 & 20.98 & 20.13 & 19.76 & 19.43 & 18.80 & 10.336 \\
\hline 7d & 38.89 & 37.95 & 36.07 & 35.68 & 34.42 & 33.11 & 31.92 & 30.64 & 29.31 & 28.53 & 1.195 \\
\hline $7 e$ & 51.16 & 50.38 & 49.11 & 48.46 & 47.56 & 47.13 & 46.28 & 45.39 & 44.21 & 43.90 & 2.349 \\
\hline $7 f$ & 64.14 & 60.28 & 58.64 & 56.72 & 54.23 & 52.17 & 50.09 & 47.21 & 45.80 & 42.38 & 0.751 \\
\hline $7 \mathrm{~g}$ & 40.06 & 38.46 & 37.71 & 34.74 & 33.24 & 32.73 & 31.29 & 29.98 & 28.39 & 27.27 & 1.473 \\
\hline $9 a$ & 65.97 & 41.46 & 40.56 & 40.2 & 38.97 & 38.05 & 37.05 & 36.38 & 35.84 & 35.26 & 13.723 \\
\hline $9 b$ & 64.99 & 62.26 & 60.68 & 56.34 & 50.12 & 46.10 & 42.01 & 41.47 & 39.42 & 38.81 & 2.414 \\
\hline $9 c$ & 67.11 & 58.80 & 54.83 & 53.61 & 50.42 & 47.02 & 44.37 & 42.60 & 41.45 & 38.13 & 0.794 \\
\hline 9d & 39.40 & 38.00 & 37.37 & 36.80 & 36.75 & 34.22 & 33.96 & 33.52 & 33.42 & 33.28 & 11.557 \\
\hline $9 e$ & 56.21 & 47.52 & 41.77 & 37.86 & 31.92 & 29.89 & 28.93 & 27.27 & 26.43 & 25.17 & 12.770 \\
\hline 9f & 38.66 & 38.22 & 36.12 & 35.80 & 35.51 & 34.78 & 34.75 & 33.86 & 32.57 & 30.64 & 112.202 \\
\hline $9 g$ & 38.14 & 36.17 & 34.74 & 33.23 & 32.82 & 31.42 & 29.23 & 28.71 & 28.02 & 27.38 & 18.345 \\
\hline $9 \mathrm{~h}$ & 47.67 & 41.55 & 38.42 & 35.17 & 34.21 & 33.76 & 32.92 & 30.64 & 29.11 & 29.02 & 1.281 \\
\hline $9 \mathbf{i}$ & 41.29 & 40.50 & 39.19 & 37.56 & 36.73 & 36.12 & 35.42 & 34.59 & 33.31 & 31.52 & 6.324 \\
\hline $\mathbf{9 j}$ & 61.43 & 56.93 & 52.13 & 49.34 & 45.14 & 43.57 & 40.13 & 37.35 & 34.64 & 30.38 & 1.361 \\
\hline ISL & 73.52 & 66.14 & 62.46 & 54.71 & 52.94 & 50.79 & 49.03 & 46.42 & 44.97 & 42.23 & 0.348 \\
\hline
\end{tabular}

${ }^{\mathrm{a}} \mathrm{CTC}_{50}$ cytotoxicity concentration $(\mu \mathrm{M})$ determined experimentally

different sensitivity to the inhibition effect of tested compounds. The results are given in Tables 3, 4 and 5 for HEK 293, BT474 and NCI-H226. Thus, from the data, it can be concluded that all test compounds are potent cytotoxic agents because of higher $\mathrm{CTC}_{50}$ at lower concentrations, and moreover, the compound $(\mathbf{4 b})\left(\mathrm{CTC}_{50}=0.922\right)$ and compound (7f) $\left(\mathrm{CTC}_{50}=0.754\right)$ were found to be most potent agent among all the compounds tested against $H E K$ 293. While compounds $(\mathbf{9 c})\left(\mathrm{CTC}_{50}=0.751\right)$ and $(\mathbf{9 j})$ $\left(\mathrm{CTC}_{50}=0.913\right)$ were found to be most potent agent among all the compounds tested against $\mathrm{BT} 474$ and $\mathrm{NCI}$ $H 226$ cell lines, respectively. But none of tested compound was found to be potent compared to standard drug indisulam. From above all cell lines such as $H E K$ 293, M468 and NCI-H226, it has been concluded that compounds (7f), $(6 \mathbf{f}),(9 \mathbf{b}),(\mathbf{9 c})$ and $(\mathbf{9 j})$ are more potent than all synthesized compounds. Compounds (6e) and (6b) have moderate activity than all synthesized compounds. Compounds (4a) and $(\mathbf{9} \mathbf{g})$ have less activity than all synthesized compounds on all cell lines. Structure activity relationship of 
Table 5 Anticancer activity (\% cytotoxicity) and $\mathrm{CTC}_{50}$ values of synthesized compounds on NCI-H226 (lung cancer cell line)

\begin{tabular}{|c|c|c|c|c|c|c|c|c|c|c|c|}
\hline \multirow[t]{4}{*}{ Treatment } & \multicolumn{10}{|c|}{$\%$ Cytotoxicity $(100-\%$ cell survival $)$ of NCI-H226 cell line at conc. $(\mu \mathrm{M})$} & \multirow[t]{4}{*}{$\mathrm{CTC}_{50}(\mu \mathrm{M})^{\mathrm{a}}$} \\
\hline & 100 & 33.33 & 11.11 & 3.7 & 1.23 & 0.41 & 0.13 & 0.045 & 0.015 & 0.005 & \\
\hline & \multicolumn{10}{|c|}{ Log conc. } & \\
\hline & 2.00 & 1.52 & 1.05 & 0.57 & 0.09 & -0.39 & -0.89 & -1.35 & -1.82 & -2.30 & \\
\hline $\mathbf{4 a}$ & 27.86 & 27.49 & 27.09 & 26.55 & 26.13 & 25.46 & 24.94 & 24.22 & 23.14 & 22.41 & 145.347 \\
\hline $4 b$ & 39.46 & 37.21 & 36.30 & 35.96 & 35.11 & 34.69 & 34.05 & 33.46 & 32.87 & 32.12 & 33.268 \\
\hline $4 c$ & 54.16 & 51.24 & 49.12 & 47.20 & 45.35 & 42.21 & 39.29 & 38.91 & 38.19 & 37.65 & 2.527 \\
\hline $4 d$ & 42.56 & 42.06 & 39.73 & 38.26 & 38.02 & 37.34 & 36.29 & 35.23 & 35.11 & 34.16 & 17.482 \\
\hline $4 e$ & 34.59 & 33.33 & 32.16 & 31.26 & 30.59 & 29.89 & 29.55 & 28.93 & 28.11 & 27.31 & 7.965 \\
\hline $4 f$ & 52.87 & 50.14 & 48.31 & 46.52 & 45.78 & 42.90 & 41.57 & 39.72 & 38.63 & 37.24 & 1.197 \\
\hline $4 g$ & 41.75 & 41.35 & 40.29 & 39.37 & 38.14 & 37.84 & 37.13 & 36.95 & 36.10 & 35.77 & 22.274 \\
\hline $6 \mathbf{a}$ & 40.92 & 38.89 & 38.22 & 36.05 & 35.61 & 33.65 & 32.94 & 32.17 & 31.57 & 30.46 & 52.953 \\
\hline $\mathbf{6 b}$ & 49.15 & 47.06 & 45.27 & 43.36 & 42.66 & 41.98 & 39.12 & 38.44 & 37.26 & 36.29 & 1.119 \\
\hline $6 c$ & 38.98 & 38.32 & 36.52 & 35.08 & 34.91 & 34.79 & 34.15 & 33.59 & 32.75 & 30.41 & 12.829 \\
\hline $6 d$ & 49.15 & 47.26 & 45.31 & 43.41 & 41.96 & 41.18 & 39.12 & 37.05 & 36.38 & 35.51 & 1.816 \\
\hline $6 e$ & 54.52 & 51.14 & 50.83 & 49.22 & 48.64 & 47.65 & 45.39 & 42.38 & 41.25 & 38.76 & 1.018 \\
\hline 6f & 65.97 & 59.62 & 57.09 & 55.18 & 54.64 & 51.26 & 48.28 & 46.54 & 44.85 & 41.28 & 0.978 \\
\hline $6 \mathrm{~g}$ & 46.01 & 43.19 & 42.63 & 41.32 & 40.65 & 39.82 & 37.34 & 36.75 & 34.95 & 33.52 & 3.108 \\
\hline $7 a$ & 36.94 & 36.21 & 35.13 & 34.55 & 32.17 & 30.41 & 29.35 & 29.17 & 28.36 & 27.44 & 10.735 \\
\hline $7 b$ & 42.44 & 41.12 & 40.65 & 39.07 & 38.79 & 37.41 & 37.05 & 35.48 & 33.62 & 33.48 & 13.829 \\
\hline $7 c$ & 40.27 & 38.88 & 38.60 & 38.21 & 38.04 & 37.79 & 36.59 & 34.75 & 34.03 & 33.23 & 1.164 \\
\hline $7 d$ & 38.92 & 38.50 & 37.91 & 35.98 & 35.37 & 35.66 & 35.17 & 34.59 & 34.13 & 33.72 & 6.342 \\
\hline $7 e$ & 36.05 & 35.80 & 35.53 & 34.87 & 34.52 & 33.48 & 31.75 & 30.46 & 29.97 & 29.04 & 12.729 \\
\hline $7 f$ & 67.99 & 65.83 & 60.68 & 56.43 & 52.12 & 46.10 & 42.62 & 40.07 & 39.26 & 38.76 & 1.784 \\
\hline $7 \mathrm{~g}$ & 38.99 & 38.74 & 37.12 & 36.26 & 36.11 & 35.72 & 35.32 & 33.62 & 32.79 & 30.66 & 10.215 \\
\hline $9 a$ & 42.36 & 41.13 & 39.07 & 38.10 & 37.89 & 37.01 & 36.15 & 35.32 & 34.84 & 33.29 & 5.674 \\
\hline $9 b$ & 37.99 & 37.72 & 37.02 & 36.62 & 36.47 & 36.11 & 35.72 & 35.43 & 29.46 & 27.75 & 1.487 \\
\hline $9 c$ & 43.51 & 40.34 & 38.19 & 37.73 & 36.15 & 35.87 & 35.12 & 34.15 & 33.25 & 31.49 & 5.726 \\
\hline 9d & 53.02 & 48.22 & 47.78 & 43.14 & 41.21 & 40.59 & 38.31 & 37.46 & 36.27 & 35.65 & 2.268 \\
\hline $9 e$ & 51.36 & 49.32 & 48.22 & 47.61 & 45.79 & 43.35 & 42.54 & 41.86 & 40.27 & 39.11 & 12.763 \\
\hline 9f & 40.39 & 38.72 & 37.14 & 36.91 & 35.67 & 34.95 & 33.42 & 32.39 & 31.24 & 30.26 & 17.327 \\
\hline $9 g$ & 42.47 & 39.75 & 39.20 & 38.61 & 37.51 & 36.33 & 35.06 & 34.11 & 33.17 & 32.72 & 166.376 \\
\hline $9 \mathrm{~h}$ & 39.98 & 39.25 & 37.94 & 37.46 & 37.24 & 36.39 & 36.32 & 35.35 & 35.01 & 32.85 & 1.467 \\
\hline $9 \mathrm{i}$ & 38.66 & 38.57 & 36.72 & 35.27 & 34.95 & 34.59 & 34.14 & 33.97 & 33.92 & 33.61 & 9.215 \\
\hline $9 j$ & 52.43 & 45.35 & 42.72 & 39.13 & 37.04 & 36.06 & 35.27 & 34.62 & 33.23 & 32.98 & 0.913 \\
\hline ISL & 59.26 & 44.69 & 38.58 & 36.46 & 34.12 & 32.98 & 31.11 & 30.20 & 28.42 & 26.37 & 0.313 \\
\hline
\end{tabular}

${ }^{\mathrm{a}} \mathrm{CTC}_{50}$ cytotoxicity concentration $(\mu \mathrm{M})$ determined experimentally

compounds showed that the presence of NH linker between aryl moiety which is substituted by electron-withdrawing group and 1,3,4-thiadiazole ring has been recognized as potent anticancer agent. Substitution on phenyl ring with chloro, methoxy and nitro group gives better anticancer activity.

The order of cytotoxic activity was electron-withdrawing group on phenyl $>$ electron-donating group on phenyl $>$ phenyl.

We can conclude that electron-releasing group on phenyl ring is responsible for less activity.

\section{Conclusion}

Thiadiazoles are mesoionic system, a poly-heteroatomic system containing a five-membered heterocycle associated with a conjugation of $\mathrm{p}$ and $\pi$ electrons and distinct regions of positive and negative charges leading to highly polarizable derivatives. This distinctive characteristic allows mesoionic compounds to effectively cross-cellular membranes and interact with biological molecules in unique ways. The good liposolubility of the sulphur atom in the heterocycle might also have a positive effect on the biological activity and 
pharmacokinetic properties of thiadiazole-containing compounds. The thiadiazole ring possesses similar chemical properties to the pyrimidine ring and can be considered a bioisostere. Given that the pyrimidine structure is found in nucleobases, components of nucleotides and the building blocks of DNA and RNA, it seems likely that thiadiazole could readily interact with DNA and RNA, potentially explaining the broad and often potent activity. Furthermore, this activity against DNA suggests that thiadiazoles derivatives could potentially be used for chemical intervention at the gene level. Compounds containing thiadiazole with high potency have been reported here, and some of them displayed excellent activities against a range of tumour cells. The ability of thiadiazoles to target DNA could explain their potential anticancer activity as uncontrolled DNA replication/cell division is a hallmark of neoplastic diseases. Furthermore, the heteroatoms of the thiadiazole are able to form interactions, such as hydrogen bonds, with biological targets that include key kinases that participate in tumorigenesis, such as CA IX and XII.

The sulfonyl group of sulphonamides is similar to the carbonate ion and can competitively inhibit CAs. Compounds containing a thiadiazole, a benzene bioisostere, should also possess high inhibitory activity when bonded with a sulphamide group. From lead compound, acetazolamide, some of the most potent compounds were synthesized and evaluated several sulphonamides as inhibitors of in vitro cancer cell growth compared with selective hCA IX inhibitor, indisulam. The affinity of 1,3,4-thiadiazole for hCA increases significantly when substituted with sulphonamides connected with Schiff base. These results indicate that the thiadiazole ring has receptor-binding ability in the context of hCA IX inhibition and in the prevention of cancer associated with CA.

\section{Experimental section}

Synthetic study

Melting points were determined in one-end-open capillary tubes on a Thermonik Precision melting point apparatus (C-PMP-2, Mumbai, India) and presented without any corrections. The IR spectra $\left(\tilde{v}, \mathrm{~cm}^{-1}\right)$ were recorded in $\mathrm{KBr}$ tablets using Shimadzu FT-IR 8400s spectrophotometer. ${ }^{1} \mathrm{H}$ nuclear magnetic resonance ( $\left.{ }^{1} \mathrm{H}-\mathrm{NMR}\right)$ spectra were recorded for the compounds on Varian EM-390 apparatus by using TMS as an internal standard. ${ }^{13} \mathrm{C}$-NMR spectra were recorded for the compounds on Bruker Avance II 400 NMR Spectrometer apparatus using TMS as an internal standard, and chemical shifts are reported in ppm ( $\delta$-scale).

Elemental analysis of the obtained compounds was performed for $\mathrm{C}, \mathrm{H}, \mathrm{N}, \mathrm{S}$ using Elemental Vario EL III
Carlo Erba 1106 analyzer. The maximum percentage differences between calculated and found values for each element were within the error and amounted to $\pm 0.4 \%$. The completion of reaction and the purity of the obtained compounds were checked by TLC on aluminium oxide 60 F254 plates (Merck Co., Whitehouse Station, NJ, USA), in a $\mathrm{CHCl} 3 / \mathrm{C} 2 \mathrm{H} 5 \mathrm{OH}(3: 1, \mathrm{v} / \mathrm{v})$ solvent system. The spots were developed in iodine chamber and visualized under ultra violet lamp $(\lambda=254 \mathrm{~nm})$.

\section{General procedure}

$N$-[(5-Amino-1,3,4-thiadiazol-2-yl)sulfonyl]benzamide (4); 5-[(4-acetamido)benzene sulphonamido]-1,3,4-thiadiazol2-( $N$-benzoyl)sulphonamide (6); and 5-[(4-amino)benzene sulphonamido]-1,3,4-thiadiazol-2-( $N$-benzoyl)sulphonamide (7) were synthesized, and their physicochemical and spectral data were reported previously (Chhajed et al., 2007, 2013). The synthesis outline is depicted in Scheme 1.

\section{$N$-\{[5-(Benzylidenamino)-1,3,4-thiadiazol-2-yl]sulphonyl $\}$ benzamide $(\mathbf{9 a})$}

Offwhitecrystals (EtOH) (this compound was prepared by refuxing 5-amino-1,3,4-thiadiazol-2-[ $N$-(benzoyl)]sulphonamide (2.74 g, $0.01 \mathrm{~mol})$ (4a) and benzaldehyde (8a) $(1.06 \mathrm{~g}, 0.01 \mathrm{~mol})$ in ethanol $(20 \mathrm{~mL})$ using $2-3$ drops of sulphuric acid as catalyst, for $12 \mathrm{~h}$. Pour it with thin stream into crushed ice. It was obtained as yellowish coloured solid and recrystallized by ethanol); yield: 63\%; $\mathrm{Mp}$ : $185-187{ }^{\circ} \mathrm{C}$; UV (MeOH) $\lambda_{\max }(\log \varepsilon) 287 \mathrm{~nm} ; R_{f}=0.62$ $\left(\mathrm{CHCl}_{3} / \mathrm{EtOH}, 3 / 1\right)$; FT-IR (KBr): $v_{\max } 3,625.1,3,037.4$, 1,693.4, 1,678.7, 1,624.32, 1,598.4, 1,557.7, 1,517-1,530.9, 1,369.6, 1,290.5, 907.25, 764.44, 756.54, $694.91 \mathrm{~cm}^{-1} ;{ }^{1} \mathrm{H}-$ NMR (DMSO, $400 \mathrm{MHz}): \delta=1.257(1 \mathrm{H}, \mathrm{s},-\mathrm{CH}-), 2.134$ $\left(6 \mathrm{H}, \mathrm{m}, \mathrm{CH}-\mathrm{C}_{6} \mathrm{H}_{5}\right), 2.590\left(6 \mathrm{H}, \mathrm{m}, \mathrm{CO}-\mathrm{C}_{6} \mathrm{H}_{5}\right), 3.965(1 \mathrm{H}, \mathrm{s}$, $\mathrm{CH}=\mathrm{N}), 4.18(1 \mathrm{H}, \mathrm{s}, \mathrm{N}-\mathrm{H}), 7.664-7.685 \mathrm{ppm}(10 \mathrm{H}, \mathrm{m}, \mathrm{Ar}-$ $\mathrm{H}) ;{ }^{13} \mathrm{C}-\mathrm{NMR}$ ([D] $\left.{ }_{6} \mathrm{DMSO}, 75 \mathrm{MHz}\right): \delta=171.46(\mathrm{C}$, amide $), 168.56\left(\mathrm{C}_{2}\right.$, thiadiazole $), 166.67\left(\mathrm{C}_{5}\right.$, thiadiazole $)$, 160.68 (C, imine), $137.78\left(\mathrm{C}_{1}, \mathrm{Ar}^{\prime}-\mathrm{C}\right.$-imine $), 136.05\left(\mathrm{C}_{1}\right.$, Ar-C-amide), $134.24\left(\mathrm{C}_{4}, \mathrm{CH}-\mathrm{Ar}^{\prime}\right), 132.52\left(\mathrm{C}_{3}, \mathrm{CH}-\mathrm{Ar}\right)$, $131.71\left(\mathrm{C}_{3}, \mathrm{CH}-\mathrm{Ar}^{\prime}\right), 130.39\left(\mathrm{C}_{5}, \mathrm{CH}-\mathrm{Ar}\right), 129.29\left(\mathrm{C}_{2}, \mathrm{CH}-\right.$ $\left.\mathrm{Ar}^{\prime}\right), 129.15\left(\mathrm{C}_{6}, \mathrm{CH}-\mathrm{Ar}^{\prime}\right), 128.84\left(\mathrm{C}_{2}, \mathrm{CH}-\mathrm{Ar}\right), 128.42\left(\mathrm{C}_{6}\right.$, $\mathrm{CH}-\mathrm{Ar}), 127.34\left(\mathrm{C}_{5}, \mathrm{CH}-\mathrm{Ar}^{\prime}\right)$; EIMS m/z [M] 370.9 (100); Anal. Calcd. for $\mathrm{C}_{16} \mathrm{H}_{12} \mathrm{~N}_{4} \mathrm{O}_{3} \mathrm{~S}_{2}: \mathrm{C}, 51.60 ; \mathrm{H}, 3.25 ; \mathrm{N}, 15.04$; S, 17.22. Found: C, 51.61; H, 3.24; N, 15.05; S, 17.22.

$N$-(\{5-[(4-Chlorobenzylidene)amino]-1,3,4-thiadiazol-2yl)sulfonyl)benzamide $(\mathbf{9 b})$

Yield: $64.2 \%$ : Mp: $212-214{ }^{\circ} \mathrm{C} ; \lambda_{\max }(\log \varepsilon) 305 \mathrm{~nm}$; $R_{f}=0.65\left(\mathrm{CHCl}_{3} / \mathrm{EtOH}, 3 / 1\right)$; FT-IR $(\mathrm{KBr}): v_{\max } 3,465.3$, $3,417.47, \quad 3,148.51, \quad 1,673.2-1,668.7, \quad 1,624.32-1,598.4$, 
1,545.9, 1,538.1-1,527.4, 1,368.9-1,358.8, 1,169.9, 968.07, 848-826.5, 764.43-674.43, $764.43 \mathrm{~cm}^{-1} ;{ }^{1} \mathrm{H}-\mathrm{NMR}$ (DMSO, $400 \mathrm{MHz}): \delta=1.359(1 \mathrm{H}, \mathrm{s},-\mathrm{CH}-), 2.342(6 \mathrm{H}, \mathrm{m}, \mathrm{CH}-$ $\left.\mathrm{C}_{6} \mathrm{H}_{5}\right), 2.678\left(6 \mathrm{H}, \mathrm{m}, \mathrm{CO}-\mathrm{C}_{6} \mathrm{H}_{5}\right), 3.623(1 \mathrm{H}, \mathrm{s}, \mathrm{CH}=\mathrm{N}), 4.41$ $(1 \mathrm{H}, \mathrm{s}, \mathrm{N}-\mathrm{H}), 7.462-8.104$ (10H, m, Ar-H) 8.24- $8.362 \mathrm{ppm}$ $(1 \mathrm{H}, \mathrm{s}, \mathrm{C}(=\mathrm{O}) \mathrm{N}-\mathrm{H}) ;{ }^{13} \mathrm{C}-\mathrm{NMR}$ ([D] $\left.{ }_{6} \mathrm{DMSO}, 75 \mathrm{MHz}\right)$ : $\delta=170.64$ (C, amide), $168.41\left(\mathrm{C}_{5}\right.$, thiadiazole $), 166.58$ $\left(\mathrm{C}_{2}\right.$, thiadiazole $), 161.68(\mathrm{C}$, imine $), 136.24\left(\mathrm{C}_{4}, \mathrm{Cl}-\mathrm{C}-\mathrm{Ar}^{\prime}\right)$, 134.16 ( $\mathrm{C}_{1}, \mathrm{Ar}-\mathrm{C}$-amide $), 133.78\left(\mathrm{C}_{1}, \mathrm{Ar}^{\prime}-\mathrm{C}\right.$-imine $), 130.25$ $\left(\mathrm{C}_{4}, \mathrm{CH}-\mathrm{Ar}\right), 129.15\left(\mathrm{C}_{3}, \mathrm{CH}-\mathrm{Ar}^{\prime}\right), 129.29\left(\mathrm{C}_{5}, \mathrm{CH}-\mathrm{Ar}^{\prime}\right)$, $129.02\left(\mathrm{C}_{3}, \mathrm{CH}-\mathrm{Ar}\right), 128.97\left(\mathrm{C}_{5}, \mathrm{CH}-\mathrm{Ar}\right), 128.84\left(\mathrm{C}_{2}, \mathrm{CH}-\right.$ $\left.\mathrm{Ar}^{\prime}\right), 128.42\left(\mathrm{C}_{6}, \mathrm{CH}-\mathrm{Ar}^{\prime}\right), 127.34\left(\mathrm{C}_{2}, \mathrm{CH}-\mathrm{Ar}\right), 127.29$ $\left(\mathrm{C}_{6}, \mathrm{CH}-\mathrm{Ar}\right)$; EIMS m/z [M] 412.9 (100); Anal. calcd. for $\mathrm{C}_{16} \mathrm{H}_{11} \mathrm{~N}_{4} \mathrm{O}_{3} \mathrm{~S}_{2} \mathrm{Cl}$ : C, 47.23; H, 2.73; N, 13.77; S, 15.76. Found: C, 47.24; H, 2.72; N, 13.75; S, 15.77 .

$N-(\{5-[(2-M e t h o x y b e n z y l i d e n e)$ amino]-1,3,4-thiadiazol-2yl) sulfonyl)benzamide $(\mathbf{9 c})$

Yield: $62.8 \%$; Mp: $201-203{ }^{\circ} \mathrm{C}$; UV $(\mathrm{MeOH}) \lambda_{\max }(\log \varepsilon)$ $315 \mathrm{~nm} ; R_{f}=0.57\left(\mathrm{CHCl}_{3} / \mathrm{EtOH}, 3 / 1\right)$; FT-IR (KBr): $v_{\max }$ 3,625.4, 3,048.7, 2,915.3-2,903.2, 1,692.8, 1,681.1-1,665.4, 1,599.9-1,536.5, 1,426.5, 1,347.1, 1,290, 1,143.2-1,129.4, 930.13-923.7, 762.6-713.1, $762.6 \mathrm{~cm}^{-1}$ (thiadiazole C-N stretching); ${ }^{1} \mathrm{H}-\mathrm{NMR}$ (DMSO, $\left.400 \mathrm{MHz}\right): \delta=1.352(1 \mathrm{H}, \mathrm{s}$, $-\mathrm{CH}-), 3.134$ (1H, s, CH- $\left.{ }_{6} \mathrm{H}_{5}\right), 3.417-3.487(3 \mathrm{H}, \mathrm{m}$, $\left.-\mathrm{OCH}_{3}\right), 6.364\left(1 \mathrm{H}, \quad \mathrm{s}, \quad \mathrm{Ar}^{\prime}-\mathrm{H}_{3,5}\right), \quad 6.84-7.16 \quad(3 \mathrm{H}$, $\left.J=7.2 \mathrm{~Hz}, \mathrm{t}, \mathrm{Ar}-\mathrm{H}_{3,4,5}\right), 8.285(2 \mathrm{H}, J=2.4 \mathrm{~Hz}, \mathrm{~d}, \mathrm{Ar}-$ $\left.\mathrm{H}_{2,6}\right), 8.58 \mathrm{ppm}(1 \mathrm{H}, \mathrm{s}, \mathrm{N}-\mathrm{H})$; ${ }^{13} \mathrm{C}-\mathrm{NMR}$ ([D] $]_{6} \mathrm{DMSO}$, $75 \mathrm{MHz}): \delta=168.21(\mathrm{C}$, amide $), 164.03\left(\mathrm{C}_{2}, \mathrm{C}-\mathrm{Ar}^{\prime}-\right.$ $\left.\mathrm{OCH}_{3}\right), 163.77(\mathrm{C}$, imine $), 162.32\left(\mathrm{C}_{2}\right.$, thiadiazole $), 162.28$ $\left(\mathrm{C}_{5}\right.$, thiadiazole $), 134.25\left(\mathrm{C}_{1}, \mathrm{CH}-\mathrm{Ar}\right), 132.22\left(\mathrm{C}_{4}, \mathrm{CH}-\mathrm{Ar}\right)$, $130.76\left(\mathrm{C}_{4}, \mathrm{CH}-\mathrm{Ar}^{\prime}\right), 130.32\left(\mathrm{C}_{6}, \mathrm{CH}-\mathrm{Ar}^{\prime}\right), 128.66\left(\mathrm{C}_{3}, \mathrm{CH}-\right.$ $\mathrm{Ar}), 128.45\left(\mathrm{C}_{5}, \mathrm{CH}-\mathrm{Ar}\right), 128.23\left(\mathrm{C}_{1}, \mathrm{CH}-\mathrm{Ar}^{\prime}\right), 127.55\left(\mathrm{C}_{2}\right.$, $\mathrm{CH}-\mathrm{Ar}), 127.46\left(\mathrm{C}_{6}, \mathrm{CH}-\mathrm{Ar}\right), 120.84\left(\mathrm{C}_{3}, \mathrm{CH}-\mathrm{Ar}^{\prime}\right), 120.44$ $\left(\mathrm{C}_{5}, \mathrm{CH}-\mathrm{Ar}^{\prime}\right), 62.32$ (C, aliphatic, OCH3) ppm; EIMS m/z $[\mathrm{M}]^{+} 404.6$ (100); Anal. calcd. for $\mathrm{C}_{17} \mathrm{H}_{14} \mathrm{~N}_{4} \mathrm{O}_{4} \mathrm{~S}_{2}$ : C, 50.74; H, 3.51; N, 13.92; S, 15.93. Found: C, 50.74; H, 3.52; N, $13.95 ; \mathrm{S}, 15.92$.

$N-(\{5-[(4-M e t h o x y b e n z y l i d e n e)$ amino]-1,3,4-thiadiazol-2yl) sulfonyl)benzamide (9d)

Yield: $65.3 \%$; Mp: 215-217 ${ }^{\circ} \mathrm{C} ; \lambda_{\max }(\log \varepsilon) 287 \mathrm{~nm}$; $R_{f}=0.45\left(\mathrm{CHCl}_{3} / \mathrm{EtOH}, 3 / 1\right)$; FT-IR $(\mathrm{KBr}): v_{\max } 3,659.8-$ 3,625.4, 2,915.3-2,903.2, 2,884.5, 1,692.8, 1,681.1-1,665.4, $1,599.9-1,536.5,1,426.5,1,347.1,1,290-1,274.4,1,143.2-$ 1,013.4, 930.13-923.7, 786.79-762.6, 762.6 cm ${ }^{-1} ;{ }^{1} \mathrm{H}-\mathrm{NMR}$ (DMSO, $400 \mathrm{MHz}): \delta=3.721\left(3 \mathrm{H}, \mathrm{s},-\mathrm{OCH}_{3}\right), 6.463(2 \mathrm{H}, \mathrm{s}$, $\left.\mathrm{Ar}^{\prime}-\mathrm{H}_{3,5}\right), 7.331-7.62(5 \mathrm{H}, J=3.0 \mathrm{~Hz}, \mathrm{~d}, \mathrm{Ar}-\mathrm{H}), 8.125(3 \mathrm{H}$, $\left.\mathrm{s}, \mathrm{Ar}-\mathrm{H}_{2,6}\right), 8.24 \mathrm{ppm}(1 \mathrm{H}, \mathrm{s}, \mathrm{C}(=\mathrm{O}) \mathrm{N}-\mathrm{H}) ;{ }^{13} \mathrm{C}-\mathrm{NMR}$ ([D] $\left.{ }_{6} \mathrm{DMSO}, 75 \mathrm{MHz}\right): \delta=170.34$ (C, amide), $165.29\left(\mathrm{C}_{4}\right.$,
$\left.\mathrm{C}-\mathrm{Ar}^{\prime}-\mathrm{OCH}_{3}\right), 163.51$ (C, imine), $162.85\left(\mathrm{C}_{2}\right.$, thiadiazole $)$, $162.34\left(\mathrm{C}_{5}\right.$, thiadiazole $), 134.29\left(\mathrm{C}_{1}, \mathrm{CH}-\mathrm{Ar}\right), 134.01\left(\mathrm{C}_{4}\right.$, $\mathrm{CH}-\mathrm{Ar}), 130.49\left(\mathrm{C}_{6}, \mathrm{CH}-\mathrm{Ar}^{\prime}\right), 130.11\left(\mathrm{C}_{2}, \mathrm{CH}-\mathrm{Ar}^{\prime}\right), 128.94$ $\left(\mathrm{C}_{3}, \mathrm{CH}-\mathrm{Ar}\right), 128.22\left(\mathrm{C}_{5}, \mathrm{CH}-\mathrm{Ar}\right), 128.11\left(\mathrm{C}_{1}, \mathrm{CH}-\mathrm{Ar}^{\prime}\right)$, $127.42\left(\mathrm{C}_{2}, \mathrm{CH}-\mathrm{Ar}\right), 127.16\left(\mathrm{C}_{6}, \mathrm{CH}-\mathrm{Ar}\right), 114.33\left(\mathrm{C}_{5}, \mathrm{CH}-\right.$ $\left.\mathrm{Ar}^{\prime}\right), 114.08\left(\mathrm{C}_{3}, \mathrm{CH}-\mathrm{Ar}^{\prime}\right), 69.41(\mathrm{C}, \mathrm{OCH} 3) \mathrm{ppm}$; EIMS m/z $[\mathrm{M}]^{+} 403.9$ (100); Anal. calcd. for $\mathrm{C}_{17} \mathrm{H}_{14} \mathrm{~N}_{4} \mathrm{O}_{4} \mathrm{~S}_{2}$ : C, 50.74; H, 3.51; N, 13.92; S, 15.93. Found: C, 50.72; H, 3.52; N, 13.96; S, 15.94 .

$N-(\{5-[(4-H y d r o x y b e n z y l i d e n e)$ amino $]-1,3,4-t h i a d i a z o l-2-$ yl)sulfonyl)benzamide (9e)

Yield: $68.2 \%$; Mp: $178-180{ }^{\circ} \mathrm{C}$; UV $(\mathrm{MeOH}) \lambda_{\max }(\log \varepsilon)$ $375 \mathrm{~nm} ; R_{f}=0.59\left(\mathrm{CHCl}_{3} / \mathrm{EtOH}, 3 / 1\right)$; FT-IR $(\mathrm{KBr}): v_{\max }$ 3,769-3,719.8, 3,671.56-3,523.8, 2,884.5, 1,713.8, 1,673.7$1,665.4,1,599.9-1,549,1,454.6-1,424.2$, 1,317.8, 1,2921,174.8, 1,174.8-1,052.1, 931.21-921.7, 786.79-762.6, 761.6-725.58 $\mathrm{cm}^{-1}$; ${ }^{1} \mathrm{H}-\mathrm{NMR}$ (400 MHz, DMSO): $\delta=$ $3.569(1 \mathrm{H}, \mathrm{s}, \mathrm{CH}=\mathrm{N}), 4.684(1 \mathrm{H}, \mathrm{s},-\mathrm{OH}), 6.547-8.623(9 \mathrm{H}$, $\mathrm{m}, \quad \mathrm{Ar}-\mathrm{H}), 8.31 \mathrm{ppm}(1 \mathrm{H}, \quad \mathrm{s}, \mathrm{C}(=\mathrm{O}) \mathrm{N}-\mathrm{H}) ;{ }^{13} \mathrm{C}-\mathrm{NMR}$ ([D] $\left.{ }_{6} \mathrm{DMSO}, 75 \mathrm{MHz}\right): \delta=169.43$ (C, imine), 167.11(C, amide $), 161.32\left(\mathrm{C}_{4}, \mathrm{C}-\mathrm{Ar}^{\prime}-\mathrm{OH}\right), 161.02\left(\mathrm{C}_{2}\right.$, thiadiazole $)$, $160.98\left(\mathrm{C}_{5}\right.$, thiadiazole $), 134.52\left(\mathrm{C}_{1}, \mathrm{CH}-\mathrm{Ar}\right), 131.17\left(\mathrm{C}_{4}\right.$, $\mathrm{CH}-\mathrm{Ar}), 130.62\left(\mathrm{C}_{6}, \mathrm{CH}-\mathrm{Ar}^{\prime}\right), 130.26\left(\mathrm{C}_{2}, \mathrm{CH}-\mathrm{Ar}^{\prime}\right), 128.82$ $\left(\mathrm{C}_{3}, \mathrm{CH}-\mathrm{Ar}\right), 128.29\left(\mathrm{C}_{5}, \mathrm{CH}-\mathrm{Ar}\right), 127.34\left(\mathrm{C}_{1}, \mathrm{CH}-\mathrm{Ar}^{\prime}\right)$, $127.55\left(\mathrm{C}_{2}, \mathrm{CH}-\mathrm{Ar}\right), 127.21\left(\mathrm{C}_{6}, \mathrm{CH}-\mathrm{Ar}\right), 114.83\left(\mathrm{C}_{5}, \mathrm{CH}-\right.$ $\left.\mathrm{Ar}^{\prime}\right), 114.12\left(\mathrm{C}_{3}, \mathrm{CH}-\mathrm{Ar}^{\prime}\right)$, ppm; EIMS m/z [M] ${ }^{+} 386.6$ (100); Anal. calcd. for $\mathrm{C}_{16} \mathrm{H}_{12} \mathrm{~N}_{4} \mathrm{O}_{4} \mathrm{~S}_{2}$ : C, 49.48; H, 3.11; N, 14.42; S, 16.51. Found: C, 49.50; H, 3.12; N, 14.40; S, 16.51 .

$N-(\{5-[(2-H y d r o x y b e n z y l i d e n e)$ amino]-1,3,4-thiadiazol-2yl\}sulfonyl)benzamide (9f)

Yield: $64.6 \%$; Mp: $220-222{ }^{\circ} \mathrm{C}$; UV $(\mathrm{MeOH}) \lambda_{\max }(\log \varepsilon)$ $478 \mathrm{~nm} ; R_{f}=0.64\left(\mathrm{CHCl}_{3} / \mathrm{EtOH}, 3 / 1\right)$; FT-IR $(\mathrm{KBr}): v_{\max }$ $3,489.1, \quad 3,261.43, \quad 2,948.5-2,884.5, \quad 1,731.22-1,635.4$, $1,614.217-1,589,1,436.06-1,505.64,1,330.70,1,232.41-$ 1,093.86, 1,093.86, 974.20-841.7, 822.2-780.44, 761.6$725.58 \mathrm{~cm}^{-1}$; ${ }^{1} \mathrm{H}-\mathrm{NMR}$ (400 MHz, DMSO): $\delta=3.582(1 \mathrm{H}$, $\mathrm{s}, \mathrm{CH}=\mathrm{N}), 4.237(1 \mathrm{H}, \mathrm{s},-\mathrm{OH}), 6.413-8.548(9 \mathrm{H}, \mathrm{m}, \mathrm{Ar}-\mathrm{H})$, $8.41 \mathrm{ppm}(1 \mathrm{H}, \mathrm{s}, \mathrm{C}(=\mathrm{O}) \mathrm{N}-\mathrm{H}) ;{ }^{13} \mathrm{C}-\mathrm{NMR}$ ([D] $]_{6} \mathrm{DMSO}$, $75 \mathrm{MHz}$ ): $\delta=166.14$ (C, imine), 165.26 (C, amide), 164.21 $\left(\mathrm{C}, \mathrm{C}_{2}-\mathrm{Ar}^{\prime}-\mathrm{OH}\right), 160.72\left(\mathrm{C}_{5}\right.$, thiadiazole $), 160.19\left(\mathrm{C}_{2}\right.$, thiadiazole), $134.82\left(\mathrm{C}_{1}, \mathrm{CH}-\mathrm{Ar}\right), 132.77\left(\mathrm{C}_{4}, \mathrm{CH}-\mathrm{Ar}^{\prime}\right), 131.38$ $\left(\mathrm{C}_{4}, \mathrm{CH}-\mathrm{Ar}\right), 130.15\left(\mathrm{C}_{6}, \mathrm{CH}-\mathrm{Ar}^{\prime}\right), 128.81\left(\mathrm{C}_{3}, \mathrm{CH}-\mathrm{Ar}\right)$, $128.49\left(\mathrm{C}_{5}, \mathrm{CH}-\mathrm{Ar}\right), 128.09\left(\mathrm{C}_{5}, \mathrm{CH}-\mathrm{Ar}^{\prime}\right), 127.40\left(\mathrm{C}_{2}, \mathrm{CH}-\right.$ Ar), $127.12\left(\mathrm{C}_{6}, \mathrm{CH}-\mathrm{Ar}\right), 114.52\left(\mathrm{C}_{1}, \mathrm{CH}-\mathrm{Ar}^{\prime}\right), 114.33\left(\mathrm{C}_{3}\right.$, $\left.\mathrm{CH}-\mathrm{Ar}^{\prime}\right)$, ppm; EIMS m/z [M] 389.4 (100); Anal. calcd. for $\mathrm{C}_{16} \mathrm{H}_{12} \mathrm{~N}_{4} \mathrm{O}_{4} \mathrm{~S}_{2}$ : C, 49.48; H, 3.11; N, 14.42; S, 16.51. Found: C, 49.47; H, 3.12; N, 14.43; S, 16.52 . 
$N$-(\{5-[(4-Hydroxy-3-methoxy benzylidene)amino]-1,3,4thiadiazol-2-yl)sulfonyl)benzamide $(\mathbf{9 g})$

Yield: $64.2 \%$; Mp: $252-254{ }^{\circ} \mathrm{C}$; UV $(\mathrm{MeOH}) \lambda_{\max }(\log \varepsilon)$ $268 \mathrm{~nm} ; R_{f}=0.67\left(\mathrm{CHCl}_{3} / \mathrm{EtOH}, 3 / 1\right)$; FT-IR $(\mathrm{KBr}): v_{\max }$ $3,537.42, \quad 3,371.43, \quad 2,927.5-2,853.4, \quad 1,692.8-1,681.1$, $1,665.4-1,599.9,1,536.05-1,426.5,1,347.1-1,290,1,274.4-$ $1,182.6, \quad 1,013.4, \quad 930.13-923.7, \quad 844.17-762.6, \quad 762.6-$ $713.1 \mathrm{~cm}^{-1} ;{ }^{1} \mathrm{H}-\mathrm{NMR}(400 \mathrm{MHz}, \mathrm{DMSO}): \delta=3.069(3 \mathrm{H}$, $\left.\mathrm{s},-\mathrm{OCH}_{3}\right), 3.659(1 \mathrm{H}, \mathrm{s}, \mathrm{CH}=\mathrm{N}), 4.428(1 \mathrm{H}, \mathrm{s},-\mathrm{OH})$, $6.126-8.262(8 \mathrm{H}, \mathrm{m}, \mathrm{Ar}-\mathrm{H}), 8.523 \mathrm{ppm}(1 \mathrm{H}, \mathrm{s}, \mathrm{C}(=\mathrm{O}) \mathrm{N}-\mathrm{H})$; ${ }^{13} \mathrm{C}-\mathrm{NMR}$ ([D] $\left.{ }_{6} \mathrm{DMSO}, 75 \mathrm{MHz}\right): \delta=170.43$ (C, imine), 167.67(C, amide), $165.09\left(\mathrm{C}_{5}\right.$, thiadiazole $), 164.18\left(\mathrm{C}_{2}\right.$, thiadiazole), $154.32\left(\mathrm{C}_{3}, \mathrm{C}-\mathrm{Ar}^{\prime}-\mathrm{OCH} 3\right), 145.13\left(\mathrm{C}_{4}, \mathrm{C}-\mathrm{Ar}^{\prime}-\right.$ $\mathrm{OH}), 135.14\left(\mathrm{C}_{1}, \mathrm{CH}-\mathrm{Ar}\right), 134.02\left(\mathrm{C}_{4}, \mathrm{CH}-\mathrm{Ar}\right), 128.83\left(\mathrm{C}_{3}\right.$, $\mathrm{CH}-\mathrm{Ar}), 128.41\left(\mathrm{C}_{5}, \mathrm{CH}-\mathrm{Ar}\right), 127.34\left(\mathrm{C}_{1}, \mathrm{CH}-\mathrm{Ar}^{\prime}\right), 127.21$ $\left(\mathrm{C}_{2}, \mathrm{CH}-\mathrm{Ar}\right), 121.62\left(\mathrm{C}_{6}, \mathrm{CH}-\mathrm{Ar}^{\prime}\right), 117.61\left(\mathrm{C}_{6}, \mathrm{CH}-\mathrm{Ar}\right)$, $117.26\left(\mathrm{C}_{5}, \mathrm{CH}-\mathrm{Ar}^{\prime}\right), 114.31\left(\mathrm{C}_{2}, \mathrm{CH}-\mathrm{Ar}^{\prime}\right), 65.17(\mathrm{C}, \mathrm{Ar}-$ $\mathrm{OCH}_{3}$ ), ppm; EIMS m/z [M] ${ }^{+} 420.1$ (100); Anal. calcd. for $\mathrm{C}_{17} \mathrm{H}_{14} \mathrm{~N}_{4} \mathrm{O}_{5} \mathrm{~S}_{2}: \mathrm{C}, 48.80 ; \mathrm{H}, 3.37 ; \mathrm{N}, 13.39 ; \mathrm{S}, 15.33$. Found: C, 48.78; H, 3.38; N, 13.41; S, 15.34 .

\section{$N-[(5-\{[4-($ Dimethylamino)benzylidene $]$ amino $\}-1,3,4-$} thiadiazol-2-yl)sulfonyl]benzamide (9h)

Yield: $67.7 \%$; Mp: $236-238{ }^{\circ} \mathrm{C}$; UV (MeOH) $\lambda_{\max }(\log \varepsilon)$ $305 \mathrm{~nm} ; R_{f}=0.42\left(\mathrm{CHCl}_{3} / \mathrm{EtOH}, 3 / 1\right)$; FT-IR $(\mathrm{KBr}): v_{\max }$ $3,652.4, \quad 3,532.12, \quad 3,114.7, \quad 2,985.3-2,896.4, \quad 1,614.2-$ 1,591.4, 1,413.1, 1,238.52-1,174.7, 804.2-783.6, 743.9$719.2 \mathrm{~cm}^{-1}$; ${ }^{1} \mathrm{H}-\mathrm{NMR}$ (400 MHz, DMSO): $\delta=2.547(6 \mathrm{H}$, $\left.\mathrm{s},-\mathrm{NCH}_{3}\right), 3.956(1 \mathrm{H}, \mathrm{s}, \mathrm{CH}=\mathrm{N}), 4.114(1 \mathrm{H}, \mathrm{s}, \mathrm{N}-\mathrm{H})$, 6.466-7.824 (9H, m, Ar-H), $8.511 \mathrm{ppm}(1 \mathrm{H}, \mathrm{s}, \mathrm{C}(=\mathrm{O}) \mathrm{N}-\mathrm{H})$; ${ }^{13} \mathrm{C}-\mathrm{NMR}\left([\mathrm{D}]_{6} \mathrm{DMSO}, 75 \mathrm{MHz}\right): \delta=169.42(\mathrm{C}$, imine), 165.21 (C, amide), $162.15\left(\mathrm{C}_{2}\right.$, thiadiazole), $162.11\left(\mathrm{C}_{5}\right.$, thiadiazole $), 154.32\left(\mathrm{C}_{4}, \mathrm{C}-\mathrm{Ar}^{\prime}-\mathrm{N}\left(\mathrm{CH}_{3}\right)_{2}\right), 134.63\left(\mathrm{C}_{1}, \mathrm{CH}-\right.$ $\mathrm{Ar}), 132.46\left(\mathrm{C}_{4}, \mathrm{CH}-\mathrm{Ar}\right), 132.23\left(\mathrm{C}_{2}, \mathrm{CH}-\mathrm{Ar}^{\prime}\right), 132.18\left(\mathrm{C}_{3}\right.$, $\mathrm{CH}-\mathrm{Ar}), 131.65\left(\mathrm{C}_{6}, \mathrm{CH}-\mathrm{Ar}^{\prime}\right), 128.12\left(\mathrm{C}_{2}, \mathrm{CH}-\mathrm{Ar}\right), 128.03$ $\left(\mathrm{C}_{6}, \mathrm{CH}-\mathrm{Ar}\right), 127.37\left(\mathrm{C}_{1}, \mathrm{CH}-\mathrm{Ar}^{\prime}\right), 127.11\left(\mathrm{C}_{3}, \mathrm{CH}-\mathrm{Ar}^{\prime}\right)$, $117.52\left(\mathrm{C}_{5}, \mathrm{CH}-\mathrm{Ar}\right), 117.11\left(\mathrm{C}_{5}, \mathrm{CH}-\mathrm{Ar}^{\prime}\right), 52.84(\mathrm{C}, \mathrm{Ar}-$ $\mathrm{NCH}_{3}$, Aliphatic), 52.47 (C, Ar- $\mathrm{NCH}_{3}$, Aliphatic) ppm; EIMS $\mathrm{m} / \mathrm{z} \quad[\mathrm{M}]^{+} 415.7$ (100); Anal. calcd. for $\mathrm{C}_{19} \mathrm{H}_{18} \mathrm{~N}_{4} \mathrm{O}_{3} \mathrm{~S}_{2}$ : C, 55.06; H, 4.38; N, 13.52; S, 15.47. Found: C, 55.07; H, 4.38; N, 13.53; S, 15.46 .

\section{$N$-(\{5-[(3-Nitrobenzylidene)amino]-1,3,4-thiadiazol-2-} yl)sulfonyl)benzamide (9i)

Yield: $61.3 \%$; Mp: $258-260{ }^{\circ} \mathrm{C}$; UV $(\mathrm{MeOH}) \lambda_{\max }(\log \varepsilon)$ $352 \mathrm{~nm} ; R_{f}=0.51\left(\mathrm{CHCl}_{3} / \mathrm{EtOH}, 3 / 1\right)$; FT-IR $(\mathrm{KBr}): v_{\max }$ 3,537.9-3,427.2, 3,128.2-3,022.3, 3,075-3,007.4, 2,341.62,331.1, 1,445.8, 1,456.8-1,531.7, 827, 1,022.8-1,078.2, 713.1-619.5 $\mathrm{cm}^{-1}$; ${ }^{1} \mathrm{H}-\mathrm{NMR}$ (400 MHz, DMSO): $\delta=$ $3.239(1 \mathrm{H}, \mathrm{s}, \mathrm{CH}=\mathrm{N}), 4.751(1 \mathrm{H}, \mathrm{s},-\mathrm{OH}), 6.872-8.421$
$(9 \mathrm{H}, \mathrm{m}, \mathrm{Ar}-\mathrm{H}), 8.645 \mathrm{ppm}(1 \mathrm{H}, \mathrm{s}, \mathrm{C}(=\mathrm{O}) \mathrm{N}-\mathrm{H}) ;{ }^{13} \mathrm{C}-\mathrm{NMR}$ ([D] $\left.{ }_{6} \mathrm{DMSO}, 75 \mathrm{MHz}\right): \delta=168.27$ (C, imine), $165.61(\mathrm{C}$, amide $), 162.23\left(\mathrm{C}_{5}\right.$, thiadiazole $), 162.18\left(\mathrm{C}_{2}\right.$, thiadiazole $)$, $154.32\left(\mathrm{C}_{3}, \mathrm{C}-\mathrm{Ar}^{\prime}-\mathrm{NO}_{2}\right), 135.71\left(\mathrm{C}_{6}, \mathrm{CH}-\mathrm{Ar}^{\prime}\right), 134.67$ $\left(\mathrm{C}_{1}, \mathrm{CH}-\mathrm{Ar}^{\prime}\right), 134.46\left(\mathrm{C}_{1}, \mathrm{CH}-\mathrm{Ar}\right), 132.49\left(\mathrm{C}_{4}, \mathrm{CH}-\mathrm{Ar}\right)$, $129.37\left(\mathrm{C}_{5}, \mathrm{CH}-\mathrm{Ar}^{\prime}\right), 128.35\left(\mathrm{C}_{3}, \mathrm{CH}-\mathrm{Ar}\right), 128.22\left(\mathrm{C}_{5}\right.$, $\mathrm{CH}-\mathrm{Ar}), 126.13\left(\mathrm{C}_{4}, \mathrm{CH}-\mathrm{Ar}^{\prime}\right), 117.11\left(\mathrm{C}_{2}, \mathrm{CH}-\mathrm{Ar}^{\prime}\right)$, $116.37\left(\mathrm{C}_{2}, \mathrm{CH}-\mathrm{Ar}\right), 116.16\left(\mathrm{C}_{6}, \mathrm{CH}-\mathrm{Ar}\right) \mathrm{ppm}$; EIMS m/z $[\mathrm{M}]^{+} 416.9$ (100); Anal. calcd. for $\mathrm{C}_{16} \mathrm{H}_{11} \mathrm{~N}_{5} \mathrm{O}_{5} \mathrm{~S}_{2}$ : C, 46.04; H, 2.66; N, 16.78; S, 15.36. Found: C, 46.05; H, $2.68 ; \mathrm{N}, 16.80 ; \mathrm{S}, 15.36$.

$N-(\{5-[($ Furan-2-ylmethylidene $)$ amino]-1,3,4-thiadiazol-2$y l\}_{\text {sulfonyl)benzamide }(\boldsymbol{9 j})}$

Brownish crystals (EtOH) (this compound was prepared by refuxing 5-amino-1,3,4-thiadiazol-2-[N-(benzoyl)]sulphonamide $(2.74 \mathrm{~g}, 0.01 \mathrm{~mol})(\mathbf{4 a})$ and Furfuldehyde $(\mathbf{8 j})$ $(0.96 \mathrm{~g}, 0.01 \mathrm{~mol})$ in ethanol $(20 \mathrm{~mL})$ using $2-3$ drops of sulphuric acid as catalyst, for $7 \mathrm{~h}$. Pour it with thin stream into crushed ice. It was obtained as dark brown coloured solid and recrystallized by ethanol); Yield: $53.04 \%$; Mp: 261-263 ${ }^{\circ} \mathrm{C}$; UV (MeOH) $\lambda_{\max }(\log \varepsilon) 412 \mathrm{~nm} ; R_{f}=0.69$ $\left(\mathrm{CHCl}_{3} / \mathrm{EtOH}, 3 / 1\right)$; FT-IR (KBr): $v_{\max } 3,634.9,3,581.22$, $3,054.2,1,635.34,1,622.4-1,595.9,1,432.4,1,254.31-$ $1,197.7, \quad 824.3-776.9, \quad 741.3-711.4 \mathrm{~cm}^{-1} ; \quad{ }^{1} \mathrm{H}-\mathrm{NMR}$ (400 MHz, DMSO): $\delta=2.547\left(6 \mathrm{H}, \mathrm{s},-\mathrm{NCH}_{3}\right), 4.116(1 \mathrm{H}$, $\mathrm{s}, \mathrm{CH}=\mathrm{N}), 6.724-7.211(3 \mathrm{H}, \mathrm{m}$, furfuryl-H), 7.446-7.918 $(5 \mathrm{H}, \mathrm{m}, \mathrm{Ar}-\mathrm{H}), 8.426 \mathrm{ppm}(1 \mathrm{H}, \mathrm{s}, \mathrm{C}(=\mathrm{O}) \mathrm{N}-\mathrm{H}) ;{ }^{13} \mathrm{C}-\mathrm{NMR}$ ([D] $\left.{ }_{6} \mathrm{DMSO}, 75 \mathrm{MHz}\right): \delta=148.22(\mathrm{C}$, imine), 167.19 (C, amide), $154.32\left(\mathrm{C}_{2}, \mathrm{C}\right.$-furfuryl $), 152.13\left(\mathrm{C}_{2}\right.$, thiadiazole $)$, $150.84\left(\mathrm{C}_{5}\right.$, thiadiazole $), 135.71\left(\mathrm{C}_{5}, \mathrm{CH}\right.$-furfuryl $), 134.63$ $\left(\mathrm{C}_{1}, \mathrm{CH}-\mathrm{Ar}\right), 132.46\left(\mathrm{C}_{4}, \mathrm{CH}-\mathrm{Ar}\right), 128.12\left(\mathrm{C}_{3}, \mathrm{CH}-\mathrm{Ar}\right)$, $128.03\left(\mathrm{C}_{5}, \mathrm{CH}-\mathrm{Ar}\right), 117.11\left(\mathrm{C}_{3}, \mathrm{CH}\right.$-furfuryl $), 111.24\left(\mathrm{C}_{2}\right.$, $\mathrm{CH}-\mathrm{Ar}), 111.06\left(\mathrm{C}_{6}, \mathrm{CH}-\mathrm{Ar}\right), 106.10\left(\mathrm{C}_{4}, \mathrm{CH}-\right.$ furfuryl $)$ ppm; EIMS m/z $[\mathrm{M}]^{+} 364.3$ (100); Anal. calcd. for $\mathrm{C}_{14} \mathrm{H}_{10} \mathrm{~N}_{4} \mathrm{O}_{4} \mathrm{~S}_{2}: \mathrm{C}, 46.40 ; \mathrm{H}, 2.78 ; \mathrm{N}, 15.46 ; \mathrm{S}, 17.70$. Found: C, 46.42; H, 2.79; N, 15.45; S, 17.39 .

Pharmacological evaluation

Antioxidant and free radical scavenging activity

Total antioxidant activity The ability of the test sample to scavenge 2,2'-azinobis-(3-ethylbenzothiazoline-6-sulphonic acid) $\left(\mathrm{ABTS}^{+}\right)$radical cation was compared with 6-hydroxy2,5,7,8-tetramethylchroman-2-carboxylic acid (trolox) standard (Chang et al., 2007; Erel, 2004; Re et al., 1999). The $\mathrm{ABTS}^{+}$radical cation was pregenerated by mixing ABTS stock solution $(7 \mathrm{mM})$ with potassium persulphate (2. $45 \mathrm{mM}$ ) (final concentration) and incubating for 12-16 h in the dark at room temperature until the reaction was complete and the absorbance was stable. The absorbance 
of the $\mathrm{ABTS}^{++}$solution was equilibrated to $0.70( \pm 0.02)$ by diluting with water at room temperature, then $1 \mathrm{~mL}$ of solution was mixed with $10 \mu \mathrm{L}$ of the test sample $(0.05-$ $10 \mathrm{mg} / \mathrm{mL}$ ), and the absorbance was measured at $734 \mathrm{~nm}$ after $6 \mathrm{~min}$. All experiments were repeated three times. The percentage inhibition of absorbance was calculated and plotted as a function of the concentration of standard and sample to determine the trolox equivalent antioxidant concentration (TEAC). To calculate the TEAC, the gradient of the plot for the sample was divided by the gradient of the plot for trolox. The $\mathrm{IC}_{50}$ inhibitory concentration $(\mathrm{nM} / \mathrm{mL})$ values of tested compounds are depicted in Table 1 . The ABTS $^{+}$radical scavenging activity of the samples was expressed as

$S \%=\left[\left(A_{\text {control }}-A_{\text {sample }}\right) / A_{\text {control }}\right] \times 100$

where $A_{\text {control }}$ is the absorbance of the blank control (ABTS $^{+}$solution without test sample), and $A_{\text {sample }}$ is the absorbance of the test sample.

Lipid peroxidation inhibitory activity Egg lecithin (3 mg/ $\mathrm{mL}$ phosphate buffer, $\mathrm{pH}$ 7.4) was sonicated in an ultrasonic sonicator for $10 \mathrm{~min}$ to ensure proper liposome formation. Test samples or standard, ascorbic acid $(100 \mu \mathrm{L})$ of different concentrations (10, 20, 30, 4050 and $100 \mu \mathrm{g} /$ $\mathrm{mL}$ ) was added to liposome mixture $(1 \mathrm{~mL})$; the control was without test sample. Lipid peroxidation was induced by adding ferric chloride $(10 \mu \mathrm{L}, 400 \mathrm{mM})$ and L-ascorbic acid $(10 \mu \mathrm{L}, 200 \mathrm{mM})$. After incubation for $1 \mathrm{~h}$ at $37{ }^{\circ} \mathrm{C}$, the reaction was stopped by adding hydrochloric acid $(2 \mathrm{~mL}, 0.25 \mathrm{~N})$ containing trichloroacetic acid $(150 \mathrm{mg} /$ $\mathrm{mL})$, thiobarbituric acid $(3.75 \mathrm{mg} / \mathrm{mL})$ and butylated hydroxy anisole $(0.50 \mathrm{mg} / \mathrm{mL})$. The reaction mixture was subsequently boiled for $15 \mathrm{~min}$, cooled and centrifuged at $1,000 \mathrm{rpm}$ for $15 \mathrm{~min}$, and the absorbance of the supernatant was measured at $532 \mathrm{~nm}$ (Duh and Yen, 1997). The $\mathrm{IC}_{50}$ values of all tested compounds are reported in Table 1 . The $\%$ inhibition at different concentrations was calculated by the following formula

$\%$ Inhibition $=\left[1-\left(V_{\mathrm{t}} / V_{\mathrm{c}}\right)\right] \times 100$

where $V_{\mathrm{t}}=$ mean absorption of test compound, $V_{\mathrm{c}}=$ mean absorption of control.

The $\mathrm{IC}_{50}(\mathrm{nM} / \mathrm{mL})$ value was derived from the $\%$ inhibition at different concentrations.

DPPH radical scavenging activity Compounds of SC series were evaluated for their in vitro free radical scavenging activities by 2,2-diphenyl-1-picrylhydrazyl (DPPH) assay method (Blois, 1958; Shishoo et al., 1999; Chhajed et al., 2007). To determine the free radical scavenging activity, a method based on the reduction of a methanolic solution of the coloured DPPH radical was used. To a set of test tubes containing methanol $(3 \mathrm{~mL})$, DPPH reagent $(2 \mathrm{mg} / \mathrm{mL})(50 \mu \mathrm{L})$ was added. The initial absorbance was measured. To these test tubes, methanolic solution of different test solutions $(1 \mathrm{mg} / \mathrm{mL})$ were added $(10-50 \mu \mathrm{L})$. Ascorbic acid $(0.5 \mathrm{mg} / \mathrm{mL})$ was also added in the concentration of $10,20,30,40,50$ and $100 \mu \mathrm{L}$. After $20 \mathrm{~min}$, absorbance was recorded at $516 \mathrm{~nm}$. The experiment was performed in triplicate. The percentage reduction in absorbance was calculated from the initial and final absorbance of each solution (Dhar and Taploo, 1982). The $\mathrm{IC}_{50}(\mathrm{nM} / \mathrm{mL})$ values are shown in Table 1.

Superoxide anion radical scavenging effect Measurement of superoxide anion scavenging activity of the synthesized compound was taken based on the method described by Nishimiki et al. (1972) and slightly modified. About $1 \mathrm{~mL}$ of nitroblue tetrazolium (NBT) solution (156 $\mu \mathrm{M}$ NBT in $100 \mathrm{mM}$ phosphate buffer, $\mathrm{pH}$ 7.4), NADH solution $(1 \mathrm{~mL})$ (reduced form of $\beta$-nicotinamide adenine dinucleotide) $(468 \mu \mathrm{M}$ in $100 \mathrm{mM}$ phosphate buffer, $\mathrm{pH}$ 7.4) and sample solution $(0.1 \mathrm{~mL})$ of compounds $(10,20,30,40,50$ and $100 \mu \mathrm{g}$ ) in distilled water were mixed and the reaction started by adding phenazine methosulphate (PMS) solution $(100 \mu \mathrm{L})(60 \mu \mathrm{M}$ PMS in $100 \mathrm{mM}$ phosphate buffer, $\mathrm{pH} 7$. 4). The reaction mixture was incubated at $25^{\circ} \mathrm{C}$ for $5 \mathrm{~min}$, and the absorbance at $560 \mathrm{~nm}$ was measured against blank samples. Catechin was used as reference compound. All the experiments were performed in triplicate, and the results were averaged. The percentage of inhibition was determined by comparing the results of control and test samples. The $\mathrm{IC}_{50}(\mathrm{nM} / \mathrm{mL})$ value are depicted in Table 1 .

Nitric oxide radical scavenging effect Nitric oxide generated from sodium nitroprusside in aqueous solution at physiological $\mathrm{pH}$ interacts with oxygen to produce nitrite ions, which were measured by the Griess reaction (Marcocci et al., 1994; Green et al., 1982). Scavenger of nitric oxide competes with oxygen leading to reduced production of nitric oxide (Mondal et al., 2006). The reaction mixture ( $3 \mathrm{~mL}$ ) containing sodium nitroprusside $(10 \mathrm{mM})$ in phosphatebuffered saline (PBS) and the compounds in different concentrations $(10,20,30,40,50$ and $100 \mu \mathrm{g})$ were incubated at $25{ }^{\circ} \mathrm{C}$ for $150 \mathrm{~min}$. At every 30 -min interval, the incubated sample $(0.5 \mathrm{~mL})$ was removed and Griess reagent $(1 \%$ sulphanilamide, $0.1 \%$ naphthylethylene diamine dihydrochloride in $\left.2 \% \mathrm{H}_{3} \mathrm{PO}_{4}\right)(0.5 \mathrm{~mL})$ was added. The absorbance of the chromophore formed was measured at $546 \mathrm{~nm}$. All the analyses were performed in triplicate, and the results were averaged. The percentage inhibition of nitric oxide generated was measured by comparing the absorbance values of control and test. Curcumin was used as a reference compound. The $\mathrm{IC}_{50}(\mathrm{nM} / \mathrm{mL})$ values are reported in Table 1. 
In vitro antimitotic activity by Allium cepa (onion) meristem root model

Small bulbs (1.5-2.0 $\mathrm{cm}$ in diameter) of the common onion, A. cepa $(2 n=16)$, were purchased from vendor at a local market. Prior to initiating the test, the outer scales of the bulbs and the dry bottom plate were removed without destroying the root primordia. The roots of A. сера were grown in distilled water in Erlenmeyer flasks $(200 \mathrm{~mL}$ capacity) under laboratory conditions (dark $24{ }^{\circ} \mathrm{C}$ ). For each synthesized compound sample, after reaching a length of $3 \mathrm{~cm}( \pm 0.5 \mathrm{~cm})$, a series of six bulbs were placed in distilled water ( $\mathrm{pH} 7.3)$ for $48 \mathrm{~h}$ and then onion roots were treated with the synthesized compound at $1 \mathrm{mg} / \mathrm{mL}$ concentrations of each tested compound. The test tubes were kept in an incubator at $22 \pm 1{ }^{\circ} \mathrm{C}$, and the test samples were changed daily at the same time. Several of the newly formed root tips were then cut from each bulb and examined for any visible morphological abnormalities. The bulbs with satisfactory root lengths $(2-2.5 \mathrm{~cm})$ were used in the study, while those with exceptionally long or short roots were discarded (on average $2-3$ bulbs). Therefore, individual sets of five bulbs were used for each extract sample.

Distilled water ( $\mathrm{pH} 7.3)$ was used as a negative control, and EMS $\left(2 \times 10^{-2} \mathrm{M}\right)$ used as a positive control mutagen (Fiskesjo, 1993, 1997). After $24 \mathrm{~h}$ of exposure, several root tips were removed from the bulbs, fixed in 3:1 (v/v) ethanol $(90 \%) /$ glacial acetic acid $(45 \%)$ and stored overnight at $4{ }^{\circ} \mathrm{C}$. The next day, they were placed in $70 \%(\mathrm{v} / \mathrm{v})$ aqueous alcohol and refrigerated until used. Allium roots were softened by digesting with $\mathrm{HCl}$ and rinsed the roots in water. After removing the water from the third rinse, the roots were covered with the orcein acetate stain. The roots were incubated in the stain for $12 \mathrm{~min}$. During this time, the very tip of the root begins to turn red as the DNA stains the numerous small actively dividing cells at the tip. A root was transferred to the centre of a clean microscope slide, and a drop of water was added. Using a razor blade most of the unstained part of the root was cut off and discarded. The root tip was covered with a cover slip and then carefully pushed down on the cover slide with the wooden end of a dissecting probe. Care should be taken to push hard, but do not twist or push the cover slide sideways. The root tip should spread out to a diameter about $0.5-1 \mathrm{~cm}$. Five slides were prepared per bulb.

Determination of cytotoxicity and genotoxicity The following parameters were used for the determination of cytotoxicity and genotoxicity:

(i) the mitotic index (MI) was calculated as the ratio between the number of mitotic cells and the total number of cells scored and expressed as percentage using following formula as per standard procedures.

Mitotic index $=\frac{\text { Number of dividing cells }}{\text { Total number of cells }} \times 100$

(ii) Chromatin aberrations (stickiness, breaks and polar deviation) were used as end points for the determination of cytogenetic effects, and micronuclei (MNC) were scored in interphase cells per 1,000 cells (\%o MNC) (Freshney, 2000).

(iii) The most frequent abnormalities are shown in microphotographs. After $72 \mathrm{~h}$ of exposure to the test samples, the root lengths were measured and used as an index of general toxicity. The results for mitotic index and root length are expressed as percentage of the negative and positive controls. Visible morphological modifications, such as changes in root consistency and colour as well as the presence of swelling (c-tumours), hooks or twists in the roots, were also observed.

\section{In vitro cytotoxicity activity by MTT assay method}

Cell line and culture medium The cancer cell line cultures of HEK 293 (epidermal kidney cell line), BT474 (breast cancer cell line) and NCI-H226 (lung cancer) were obtained from Pasteur Institute of India, Coonoor, India, and were cultured in RPMI-1640 and $10 \%$ heat-activated New born calf serum with antibiotics [penicillin (1,000 I. $\mathrm{U} . / \mathrm{mL})$, streptomycin $(100 \mu \mathrm{g} / \mathrm{mL})$ and amphotericin B $(25 \mu \mathrm{g} / \mathrm{mL})]$. The cells were maintained at $37^{\circ} \mathrm{C}$ in a humidified atmosphere with $5 \% \mathrm{CO}_{2}$ and were subcultured twice a week.

Determination of cytotoxicity by microculture tetrazolium (MTT) assay The monolayer cell culture $(100 \mu \mathrm{L})$ was trypsinized, and the cell count was adjusted to 3 . $0 \times 10^{5}$ cells $/ \mathrm{mL}$ using medium containing $10 \%$ new born calf serum. To each well of the 96-well microtitre plate, the diluted cell suspension (approximately 10,000 cells) $(0.1 \mathrm{~mL})$ was added and kept for $24 \mathrm{~h}$ in incubator at $37{ }^{\circ} \mathrm{C}$ in $5 \% \mathrm{CO}_{2}$ atmosphere for cell monolayer formation. After $24 \mathrm{~h}$, when a partial monolayer was formed at the bottom of the well, the supernatant was flicked off, the monolayer was washed once, and different drugs, i.e. synthesized compounds $(100 \mu \mathrm{L})$, were added to the cells in microtitre plates. The plates were then incubated at $37{ }^{\circ} \mathrm{C}$ for 3 days in $5 \% \mathrm{CO}_{2}$ atmosphere, and microscopic examination was carried out and observations recorded every $24 \mathrm{~h}$. After $72 \mathrm{~h}$, the sample solution in the wells was flicked off; MTT dye $(50 \mathrm{~mL})$ was added to each well; plates were gently shaken and incubated for $4 \mathrm{~h}$ at $37{ }^{\circ} \mathrm{C}$ in 
$5 \% \mathrm{CO}_{2}$ incubator. The supernatant was removed and propanol $(50 \mu \mathrm{L})$ was added; the plates were gently shaken to solubilize the formed formazan. The absorbance was measured using a microplate reader at a wavelength of $490 \mathrm{~nm}$ (Edmondson et al., 1988; Prasad et al., 2005; Chiruvella et al., 2008; Chang et al., 2007).

Acknowledgments The authors are highly thankful to Director, Sophisticated Analytical Instrumentation Facility (SAIF), Panjab University, Chandigarh; Department of Chemistry, Pune University, Pune, and Director, Sophisticated Analytical Instrumental Laboratory (SAIL), School of Pharmaceutical Sciences, Rajiv Gandhi Proudyogiki Viswavidyalaya, Bhopal for providing for providing the necessary spectral analysis facilities to carry out this research work.

Conflict of interest The authors declare no conflict of interest.

Open Access This article is distributed under the terms of the Creative Commons Attribution License which permits any use, distribution, and reproduction in any medium, provided the original author(s) and the source are credited.

\section{References}

Abrahum DJ (2003) Burger's medicinal chemistry and drug discovery: principle and practice, vol 1, 6th edn. Wiley, New York

Angayarkanni J, Ramkumar KM, Poornima T, Priyadarshini U (2007) Cytotoxic activity of Amorphophallus paeoniifolius tuber extract in vitro. Am Eurasian J Agri Environ Sci 2(4):395-398

Ashida S, Nishimori I, Tanimura M, Onishi S, Shuin T (2002) Effects of von Hippel-Lindau gene mutation and methylation status on expression of transmembrane carbonic anhydrases in renal cell carcinoma. J Cancer Res Clin Oncol 128(10):561-568

Auti S, Pagare R, Ahire D, Sawale V (2010) Cytogenetical studies on the effect of omnacortil on root tip cells of Allium cepa L. J Cell Tissue Res 10(3):2331-2335

Blois MS (1958) Antioxidant determination by the use of a stable free radical. Nature 181(4617):1199-1200. doi:10.1038/1811199a0

Brackett CC, Singh H, Block JH (2004) Likelihood and mechanisms of cross-allergenicity between sulfonamide antibiotics and other drugs containing a sulfonamide functional group. Pharmacotherapy 24(7):856-870

Brzozowski Z, Slawinski J, Saczewski F, Innocenti A, Supuran CT (2010) Carbonic anhydrase inhibitors: synthesis and inhibition of the human cytosolic isozymes I and II and transmembrane isozymes IX, XII (cancer-associated) and XIV with 4-substituted 3-pyridinesulfonamides. Eur J Med Chem 45(6):2396-2404

Budavari S (1989) The Merck Index: an encyclopedia of chemicals, drugs, and biologicals, 11th edn. Merck Research Laboratories, Whitehouse Station, p 3782

Cecchi A, Hulikova A, Pastorek J, Pastorekova S, Scozzafava A, Winum JY, Montero JL, Supuran CT (2005) Carbonic anhydrase inhibitors. Design of fluorescent sulfonamides as probes of tumour-associated carbonic anhydrase IX that inhibit isozyme IX-mediated acidification of hypoxic tumours. J Med Chem 48(15):4834-4841

Chang HY, Ho YL, Sheu MJ, Lin YH, Tseng MC, Wu SH, Huang GJ, Chang YS (2007) Antioxidant and free radical scavenging activities of Phellinus merrillii extracts. Bot Stud 48:407-417

Chegwidden WR, Spencer IM, Supuran CT (2001) The roles of carbonic anhydrase in cancer. In: Xue G, Xue Y, Xu Z, Hammond GL, Lim AH (eds) Gene families: studies of DNA,
RNA, enzymes, and proteins. World Scientific, Singapore, pp 157-169

Chhajed MR, Khedekar PB, Mundhey AS (2007) Synthesis and free radical scavenging activity of some 1,3,4-thiazole derivatives. Indian J Heterocycl Chem 16:259-262

Chhajed MR, Shrivastava AK, Taile VS (2013) Design and syntheses of some new 5-[benzene sulphonamido]-1,3,4-thiadiazol-2sulphonamide as potent antiepileptic agent. Macroheterocycles 6(2):199-209. doi:10.6060/mhc130116c

Chiruvella KK, Kari V, Choudhary B, Nambiar M, Ghanta RG, Raghavan SC (2008) Methyl angolensate, a natural tetranortriterpenoid induces intrinsic apoptotic pathway in leukemic cells. FEBS Lett 582(29):4066-4076. doi:10.1016/j.febslet.2008.11.001

Desai NC, Shukla HK, Astik RR, Thaker KA (1984) Studies on some thiosemicarbazones and 1,3,4-thiadiazolines as potential antitubercular and antibacterial agents. J Indian Chem Soc LXI: $168-196$

Dhar DN, Taploo CL (1982) Schiff bases and their applications. J Sci Ind Res 41:501-506

Duh PD, Yen GH (1997) Antioxidative activity of three herbal water extracts. Food Chem 60:639-645

Edmondson JM, Armstrong LS, Martinez AO (1988) A rapid and simple MTT-based spectrophotometric assay for determining drug sensitivity in monolayer cultures. J Tissue Cult Methods $11: 15-17$

Erel O (2004) A novel automated direct measurement method for total antioxidant capacity using a new generation, more stable ABTS radical cation. Clin Biochem 37(4):277-285

Eroglu E (2008) Some QSAR studies for a group of sulfonamide Schiff base as carbonic anhydrase CA II inhibitors. Int J Mol Sci 9:181-197

Fiskesjo G (1993) Allium test I: a 2-3 day plant test for toxicity assessment by measuring the mean root growth of onions (allium cepa L.). Environ Toxicol Water Qual 8(4):461-470. doi:10. 1002/tox.2530080410

Fiskesjo G (1997) Allium test for screening chemicals; Evaluation of cytological parameters. In: Wang W, Gorsuch JW, Hughes JS (eds) Plants for environmental studies. CRC Lewis Publishers, New York, pp 308-333

Freshney RI (2000) Cytotoxicity. In: Liss AR (ed) Cultures of animal cells, a manual of basic technique. Wiley, New York

Fujikawa-Adachi K, Nishimori I, Taguchi T, Onishi S (1999) Human carbonic anhydrase XIV (CA14): cDNA cloning, mRNA expression, and mapping to chromosome 1. Genomics 61(1):74-81

Green LC, Wagner DA, Glogowski J, Skipper PL, Wishnok JK, Tannenbaum SR (1982) Analysis of nitrate, nitrite, and [15N]nitrate in biological fluids. Anal Biochem 126(1):131-138

Gupta A, Mishra P, Kashaw SK, Jatav V, Stables JP (2008) Synthesis of 3-aryl amino/amino-4-aryl-5-imino-D2-1,2,4-thiadiazoline and evaluated for anticonvulsant activity. Eur J Med Chem 43(4):749-754

Hanna MA, Girges MM, Rasala D, Gawinecki R (1995) Synthesis and pharmacological evaluation of some novel 5-(pyrazol-3-yl)thiadiazole and oxadiazole derivatives as potential hypoglycemic agents. Arzneim-Forsch- Drug Res 45(10):1074-1078

Harrison TR (1994) Harrison's principles of internal medicine, 13th edn. McGraw-Hill, New Delhi, p 604

Jatav V, Mishra P, Kashaw S, Stables JP (2008) CNS depressant and anticonvulsant activities of some novel 3-[5-substituted-1,3,4thiadiazole-2-yl]-2-styryl quinazoline-4(3H)-ones. Eur J Med Chem 43(9):1945-1954

Kamb A (2005) Opinion: what's wrong with our cancer models? Nat Rev Drug Discov 4(2):161-165

Kaunisto K, Parkkila S, Rajaniemi H, Waheed A, Grubb J, Sly WS (2002) Carbonic anhydrase XIV: luminal expression suggests key role in renal acidification. Kidney Int 61(6):2111-2118 
Khan SA, Siddiqui AA, Shibeer B (2002) Analgesic activity of isatin derivatives. Asian J Chem 14:1117-1118

Kumar A, Shrivastava VK, Archana (2003) Synthesis of newer indolyl thiadiazoles and their thiazolidinones and formazans as potential anticonvulsant agents. Indian $\mathrm{J}$ Pharm Sci 65(4):358-362

Kumar D, Kumar MN, Chang KH, Shah K (2010) Synthesis and anticancer activity of 5-(3-indolyl)-1,3,4-thiadiazoles. Eur J Med Chem 45(10):4664-4668

Kuzmin VE, Artemenko AG, Lozytska RN, Fedtchouk AS, Lozitsky VP, Muratov EN, Mescheriakov AK (2005) Investigation of anticancer activity of macrocyclic Schiff bases by means of 4DQSAR based on simplex representation of molecular structure. Environ Res 16(3):219-230

Manrao MR, Kaur B, Shrma RC, Kalsi PS (1982) Reaction of active methylene compounds with veratraldehyde Schiff bases and antifungal activity of products. Ind J Chem 21:1054-1060

Manrao MR, Singh B, Shrma JR, Kalsi PS (1995) Effect o hydroxyl group on antifungal activity of Schiff bases. Pestic Res J $7: 157-159$

Manrao MR, Goel M, Shrma JR (2001) Synthesis and fungitoxicity of ketimines of acetophenone. Ind J Agric Chem 34:86-88

Marcocci L, Maguire JJ, Droy-Lefaix MT, Packer L (1994) The nitric oxide scavenging property of Ginkgo biloba extract EGb 761. Biochem Biophys Res Comm 201(2):748-755

Miller NJ, Rice-Evans CA (1994) Total antioxidant status in plasma and body fluids. Methods Enzymol 234:279-293

Miller NJ, Rice-Evans CA (1996) Spectrophotometric determination of antioxidant activity. Redox Rep 2:161-171

Minchinton AI, Tannock IF (2006) Drug penetration in solid tumours. Nat Rev Cancer 6(8):583-592

Mondal SK, Chakraborty G, Gupta M, Muzumdar UK (2006) In vitro antioxidant activity of Diospyros malabarika kostel bark. Indian J Exp Biol 44:39-44

More SV, Dongarkhadekar DV, Chavan RN, Jadhav WW, Bhusare SR, Pawar RP (2002) Synthesis and antibacterial activity of new Schiff bases, 4-thiazolidinones and 2-azetidinones. J Ind Chem Soc 79:768-769

Nishimiki M, Rao NA, Yagi K (1972) The occurrence of superoxide anion in the reaction of reduced phenazine methosulphate and molecular oxygen. Biochem Biophys Res Comm 46(2):849-853

Noolvi MN, Patel HM, Singh N, Gadad AK, Cameotra SS, Badiger A (2011) Synthesis and anticancer evaluation of novel 2-cyclopropylimidazo[2,1-b][1,3,4]-thiadiazole derivatives. Eur J Med Chem 46(9):4411-4418

Oruc EE, Rollas S, Kandemirli F, Shvets N, Dimoglo AS (2004) 1,3,4-Thiadiazole derivatives. Synthesis, structure elucidation, and structure-antituberculosis activity relationship investigation. J Med Chem 47:6760-6767

Pacheco H, Correnberger L, Pillon D, Thiolliere JT (1970) Chem Abstr 72:111001-111002

Pandey VK, Tusi S, Tusi Z, Raghubir R, Dixit M, Joshi MN, Bajpai SK (2004) Thiadiazolyl quinazolones as potential antiviral and antihypertensive agents. Indian J Chem 43B:180-183

Parkkila S, Rajaniemi H, Parkkila AK, Kivelä J, Waheed A, Pastorekova S, Pastorek J, Sly WS (2000) Carbonic anhydrase inhibitor suppresses invasion of renal cancer cells in vitro. Proc Natl Acad Sci USA 97:2220-2224

Parkkila S, Parkkila AK, Rajaniemi H, Shah GN, Grubb JH, Waheed A, Sly WS (2001) Expression of membrane-associated carbonic anhydrase XIV on neurons and axons in mouse and human brain. Proc Natl Acad Sci USA 98(4):1918-1923

Parkkila S, Kivela AJ, Kaunisto K, Parkkila AK, Hakkola J, Rajaniemi H, Waheed A, Sly WS (2002) The plasma membrane carbonic anhydrase in murine hepatocytes identified as isozyme XIV. BMC Gastroenterol 2:13. doi:10.1186/1471-230X-2-13
Pastorek J, Pastorekova S, Callebaut I, Marnon JP, Zelnik V, Opavsky R, Zatovicova M, Liao S, Portetelle D, Stanbridge EJ, Zavada J, Burny A, Kettmann R (1994) Cloning and characterization of $\mathrm{MN}$, a human tumor-associated protein with a domain homologous to carbonic anhydrase and a putative helix-loop-helix DNA binding segment. Oncogene 9(10):2877-2888

Pastorekova S, Parkkila S, Parkkila AK, Opavsky R, Zelnik V, Saarnio J, Pastorek J (1997) Carbonic anhydrase IX, MN/CA IX: analysis of stomach complementary DNA sequence and expression in human and rat alimentary tracts. Gastroenterology 112(2):398-408

Patil R, Biradar JS (2001) Synthesis and pharmacological evaluation of Substituted-2-triazolo(3,4-b)[1,3,4,]-thiadiazoles. Indian J Pharm Sci 63(4):299-305

Pattan SR, Kekare P, Dighe NS, Nirmal SA, Musmade DS, Parjane SK, Daithankar AV (2009) Synthesis and biological evaluation of some 1,3,4-thiadiazoles. J Chem Pharm Res 1(1):191-198

Pavlica M, Besendorfer V, Rosa J, Papes D (2000) The cytotoxic effect of wastewater from the phosphoric gypsum depot on common oak (Quercus robur L.) and shallot (Allium cepa var. ascalonicum). Chemosphere 41(10):1519-1527

Prasad KN, Ashok G, Raghu C, Shivamurthy GR, Vijayan P, Ardhya SM (2005) In vitro cytotoxic properties of Ipomoea aquatica leaf. Indian J Pharmacol 37(6):397-398. doi:10.4103/0253-7613. 19079

Rathelot P, Azas N, El-Kashef H, Delmas F, Di Giorgio C, TimonDavid P, Maldonado J, Venelle P (2002) 1,3-Diphenylpyrazoles: synthesis and antiparasitic activities of azomethine derivatives. Eur J Med Chem 37(8):671-679

Re R, Pellegrini N, Proteggente A, Pannala A, Yang M, Rice-Evans C (1999) Antioxidant activity applying an improved ABTS radical cation decolorization assay. Free Radic Biol Med 26(9/ 10): $1231-1237$

Salimon J, Salih N, Yousif E, Hameed A, Ibraheem H (2010) Synthesis and antibacterial activity of some new 1,3,4-oxadiazole and 1,3,4-thiadiazole derivatives. Aus J Basic Appl Sci 4(7):2016-2021

Sega GA (1984) A review of the genetic effects of ethyl methanesulfonate. Mutat Res 134(2-3):113-142

Sharma KP, Jolly VS, Pathak P (1998) Schiff base and their derivatives as potential anticancer agents. Ultra Sci Phys Sci 10:263-266

Sharma R, Talesara GL, Nagda DP (2006) Synthesis of various isoniazidothiazolidinones and their imidoxy derivatives of potential biological interest. Arkivoc i:1-12

Sharma R, Sainy J, Chatuvedi SC (2008) 2-Amino-5-sulfanyl-1,3,4thiadiazoles: a new series of selective cyclooxygenase-2 inhibitors. Acta Pharm 58(3):317-326

Shishoo CJ, Ravikumar T, Jain KS, Rathod IS, Gandhi TP, Satia MC (1999) Synthesis of novel 1,2-(un)substituted-3-amino-5-aryl-6arylaminopyrazolo[3,4-d]pyrimidin-4(5H)-ones and their biological activities. Indian J Chem 38(9):1075-1085

Shrivastava SK, Shrivastava S, Shrivastava SD (1999) Synthesis of new carbazolyl-thiadiazole-2-oxoazetidines: antimicrobial, anticonvulsant and anti-inflammatory agents. Indian J Chem 38B: $183-187$

Slatore CG, Tilles SA (2004) Sulfonamide hypersensitivity. Immunol Allergy Clin N Am 24(3):477-490

Stillings MR, Welbourn AP, Walter DS (1986) Substituted 1,3,4thiadiazoles with anticonvulsant activity. 2. Aminoalkyl derivatives. J Med Chem 29:2280-2284

Supran CT, Barboiu M, Luca C, Pop E, Brewster ME, Dinculescu A (1996) Carbonic anhydrase activators, part 4, synthesis of mono and bis pyridinium salt derivatives of 2-amino-5-(2-aminoethyl)and 2-amino-5-(3aminopropyl)-1,3.4-thiazole and their Interaction with isoenzyme (II). Eur J Med Chem 31:597-606 
Supran CT, Scozzafava A, Casini A (2003) Carbonic anhydrase inhibitors. Med Res Rev 23(2):146-189

Supuran CT (2008) Carbonic anhydrases: novel therapeutic applications for inhibitors and activators. Nat Rev Drug Discov 7(2):168-181

Supuran CT, Scozzafava A (2000) Carbonic anhydrase inhibitors and their therapeutic potential. Expert Opin Ther Pat 10:575-600

Supuran CT, Scozzafava A, Conway I (2004) Carbonic anhydrase, its inhibitors and activators. CRC, New York, pp 1-363

Svastova E, Hulikova A, Rafajova M, Zatovicova M, Gibadulinova A, Casini A, Cecchi A, Scozzafava A, Supuran CT, Pastorek J, Pastorekova S (2004) Hypoxia activates the capacity of tumourassociated carbonic anhydrase IX to acidify extracellular $\mathrm{pH}$. FEBS Lett 577(3):439-445

Taggi AE, Hafez AM, Wack H, Young B, Lectka D (2002) The development of the first catalyzed reaction of ketenes and imines: catalytic, asymmetric synthesis of $\beta$-lactams. J Am Chem Soc 124:6626-6635

Tilles SA (2001) Practical issues in the management of hypersensitivity reactions: sulfonamides. South Med J 94(8):817-824

Tureci O, Sahin U, Vollmar E, Siemer S, Gottert E, Seitz G, Parkkila AK, Shah GN, Grubb JH, Pfreundschuh M, Sly WS (1998) Human carbonic anhydrase XII: cDNA cloning, expression, and chromosomal localization of a carbonic anhydrase gene that is overexpressed in some renal cell cancers. Proc Natl Acad Sci USA 95(13):7608-7613. doi:10.1073/pnas.95.13.7608

Vaghasiya YK, Nair RS, Baluja M, Chanda S (2004) Synthesis, structural determination and antibacterial activity of compounds derived from vanillin and 4-aminoantipyrine. J Serb Chem Soc 69:991-998

Varandas LS, Fraga CAM, Miranda ALP, Barreiro EJ (2005) Design, synthesis and pharmacological evaluation of new nonsteroidal anti-inflammatory 1,3,4-thiadiazole derivatives. Lett Drug Des Discov 2(1):62-67

Verma M, Pandya SN, Singh KN, Stables JP (2004) Anticonvulsant activity of Schiff bases of isatin derivatives. Acta Pharm 54(1):49-56

Wilson CO, Gisvold O (1991) Anti-infective agents, antibacterial antibiotics. In: Swarbrick EA (ed) Textbook of organic medicinal and pharmaceutical chemistry, 9th edn. Wiley, New York

Wykoff CC, Beasley JN, Watson PH, Turner KJ, Pastorek J, Sibtain A, Wilson DG, Turley H, Talks KL, Maxwell HP, Pugh WC, Ratcliffe JP, Harris LA (2000) Hypoxia-inducible expression of tumor-associated carbonic anhydrases. Cancer Res 60:7075-7083

Zamani K, Faghihi K, Tofighi T, Shariatzadeh MR (2004) Synthesis and antimicrobial activity of some pyridyl and naphthyl substituted 1,2,4-triazole and 1,3,4-thiadiazole derivatives. Turk J Chem 28:95-100 